\title{
Bulk-driven acoustic streaming at resonance in closed microcavities
}

\author{
Bach, Jacob Søberg; Bruus, Henrik
}

Published in:

Physical Review E

Link to article, DOI:

10.1103/PhysRevE.100.023104

Publication date:

2019

Document Version

Publisher's PDF, also known as Version of record

Link back to DTU Orbit

Citation (APA):

Bach, J. S., \& Bruus, H. (2019). Bulk-driven acoustic streaming at resonance in closed microcavities. Physical Review E, 100(2), [023104]. https://doi.org/10.1103/PhysRevE.100.023104

\section{General rights}

Copyright and moral rights for the publications made accessible in the public portal are retained by the authors and/or other copyright owners and it is a condition of accessing publications that users recognise and abide by the legal requirements associated with these rights.

- Users may download and print one copy of any publication from the public portal for the purpose of private study or research.

- You may not further distribute the material or use it for any profit-making activity or commercial gain

- You may freely distribute the URL identifying the publication in the public portal

If you believe that this document breaches copyright please contact us providing details, and we will remove access to the work immediately and investigate your claim 


\title{
Bulk-driven acoustic streaming at resonance in closed microcavities
}

\author{
Jacob S. Bach* and Henrik Bruus $\odot^{\dagger}$ \\ Department of Physics, Technical University of Denmark, DTU Physics Building 309, DK-2800 Kongens Lyngby, Denmark
}

(Received 22 May 2019; published 7 August 2019)

\begin{abstract}
Bulk-driven acoustic (Eckart) streaming is the steady flow resulting from the time-averaged acoustic energy flux density in the bulk of a viscous fluid. In simple cases, like the one-dimensional single standing-wave resonance, this energy flux is negligible, and therefore the bulk-driven streaming is often ignored relative to the boundary-driven (Rayleigh) streaming in the analysis of resonating acoustofluidic devices with length scales comparable to the acoustic wavelength. However, in closed acoustic microcavities with viscous dissipation, two overlapping resonances may be excited at the same frequency as a double mode. In contrast to single modes, the double modes can support a steady rotating acoustic energy flux density and thus a corresponding rotating bulk-driven acoustic streaming. We derive analytical solutions for the double modes in a rectangular-box-shaped cavity including the viscous boundary layers, and use them to map out possible rotating patterns of bulk-driven acoustic streaming. Remarkably, the rotating bulk-driven streaming may be excited by a nonrotating actuation, and we determine the optimal geometry that maximizes this excitation. In the optimal geometry, we finally simulate a horizontal $2 \times 2,4 \times 4$, and $6 \times 6$ streaming-roll pattern in a shallow square cavity. We find that the high-frequency $6 \times 6$ streaming-roll pattern is dominated by the bulk-driven streaming as opposed to the low-frequency $2 \times 2$ streaming pattern, which is dominated by the boundary-driven streaming.
\end{abstract}

DOI: 10.1103/PhysRevE.100.023104

\section{INTRODUCTION}

Acoustophoresis, particle migration by ultrasound in a microfluidic setting, is an increasingly popular method for noncontact and label-free handling of microparticles suspended in a fluid. Examples include acoustic separation [1-3], trapping [4,5], and tweezing [6-8], as well as enrichment of cancer cells $[9,10]$ and bacteria $[11,12]$, and size-independent sorting of cells [13].

The acoustic field induces the acoustic radiation force that acts on suspended particles and scales with the particle volume [14-18], as well as the acoustic streaming in the fluid [19-22] that gives rise to a viscous Stokes drag force on the particles which scales with the linear size of the particles [23]. Consequently, the streaming-induced drag force is the dominating acoustic force for particles smaller than a critical size. For example, for an aqueous suspension of spherical polystyrene particles in a 1-MHz ultrasound field, the critical radius has been determined to be around $2 \mu \mathrm{m}[24,25]$. To extend the application of acoustophoresis into the regime of submicrometer particles, such as bacteria, viruses, and exosomes, a deep understanding of acoustic streaming is therefore important.

The first theoretical analysis of acoustic streaming dates back to Lord Rayleigh [19], who investigated the streaming generated by the viscous boundary layers close to the walls in planar and cylindrical systems. This analysis was later generalized by Nyborg [22], who formulated a slip velocity condition for the acoustic streaming outside the viscous

\footnotetext{
*jasoba@fysik.dtu.dk

†bruus@fysik.dtu.dk
}

boundary layers at weakly curved boundaries oscillating in the normal direction. Further extensions to this slip-velocity theory involve curvilinear corrections for curved boundary [26], general motion of a flat boundary [27], and general motion of a curved boundary [28]. This kind of streaming is caused by the velocity mismatch between the boundary and the acoustic wave in the bulk, and we refer to it as boundarydriven acoustic streaming.

Acoustic streaming can also be generated by attenuation of the acoustic wave in the bulk as shown by Eckart [29], who established the basic theory and investigated the steady flow caused by a sound beam. This kind of streaming is generated by a force, which is proportional to the acoustic energy flux density (or intensity) such that, say, a sound beam generates acoustic streaming in its direction of propagation. We refer to this kind of streaming as bulk-driven acoustic streaming.

While bulk-driven streaming is known to have a clear effect in systems much larger than the acoustic wavelength [30], it is often neglected in studies of resonating devices with length scales comparable with the acoustic wavelength. However, the bulk-driven streaming was taken into account implicitly by Antfolk et al. in their study of a long straight microchannel with a nearly square cross section [11], as they made a direct numerical simulation of the acoustofluidic properties of the system. They showed numerically that the experimentally observed single-vortex streaming pattern, which remarkably appeared in their microchannel instead of the usual quadrupolar Rayleigh-vortex pattern, could be explained if two orthogonal acoustic resonances with nearly identical resonance frequencies were excited with a quarter-period phase difference at the same frequency.

Such orthogonal phase-shifted acoustic waves have been used in other experiments to generate an acoustic radiation 
torque acting on suspended particles [26,31-35]. Also traveling Bessel beams [36,37] as well as arrays of holographic acoustic elements [38] have been used for this purpose, but not for generating specific streaming patterns.

Inspired by the work of Antfolk et al. [11], we study in this paper bulk-driven acoustic streaming at resonance in rectangular-box-shaped cavities with acoustically hard walls and side lengths comparable to the acoustic wavelength. The paper is organized as follows: In Sec. II we present the governing equations for the acoustic pressure and the acoustic streaming at resonance, where the viscous boundary layers are taken into account using our recent boundary-layer analysis [28]. We apply these equations in Sec. III to derive analytical solutions for the acoustic single-mode pressure resonances in the rectangular cavity. We also introduce the so-called doublemode resonances, where two overlapping single modes are excited simultaneously at the same frequency. In Sec. IV we establish the so-called overlap and phase conditions for double-mode resonances that lead to a strong acoustic body force, which causes the bulk-driven streaming. A main result is presented in Sec. V, where we show how a weak symmetry breaking of a perfect square cavity can lead to a rotating body force even for a nonrotating actuation. We validate our theory in Sec. VI by direct numerical simulation in the case of a shallow nearly square cavity similar to the geometry studied experimentally by Hagsäter et al. [39]. We predict that the bulk-driven streaming in closed microcavities is enhanced by increasing the frequency and the bulk viscosity, just as is the case for Eckart streaming in open systems [29]. We propose to test this prediction by replacing water with pyridine in the microcavity. Finally, we discuss our results in Sec. VII and present our conclusions in Sec. VIII.

\section{GOVERNING EQUATIONS}

The time-harmonic acoustic fields in the fluid domain $\Omega$ are induced by the time-harmonic displacement field $\boldsymbol{u}(\boldsymbol{r}, t)$ of the wall at the boundary $\partial \Omega$ of the domain,

$$
\boldsymbol{u}(\boldsymbol{r}, t)=\operatorname{Re}\left[\boldsymbol{u}_{1}(\boldsymbol{r}) e^{-i \omega t}\right], \quad \boldsymbol{r} \in \partial \Omega,
$$

where $\boldsymbol{r}$ is position, $t$ is time, $\omega=2 \pi f$ is the angular frequency corresponding to the driving frequency $f$, and "Re" is the real part of complex-valued fields. In the quiescent and homogeneous fluid of mass density $\rho_{\mathrm{fl}}$ and ambient pressure $p_{\mathrm{f}}$, we apply standard perturbation theory in the small Mach number $\mathrm{Ma}=\frac{v_{1}}{c_{\mathrm{f}}} \sim \frac{\rho_{1}}{\rho_{\mathrm{f}}} \ll 1$ (see, e.g., Ref. [18]) to describe the fluid velocity $v(r, t)$, pressure $p(\boldsymbol{r}, t)$, and mass density $\rho(\boldsymbol{r}, t)$ :

$$
\begin{aligned}
& p(\boldsymbol{r}, t)=p_{\mathrm{fl}}+\operatorname{Re}\left[p_{1}(\boldsymbol{r}) e^{-i \omega t}\right]+p_{2}(\boldsymbol{r}), \\
& \boldsymbol{v}(\boldsymbol{r}, t)=\mathbf{0}+\operatorname{Re}\left[\boldsymbol{v}_{1}(\boldsymbol{r}) e^{-i \omega t}\right]+\boldsymbol{v}_{2}(\boldsymbol{r}), \\
& \rho(\boldsymbol{r}, t)=\rho_{\mathrm{fl}}+\operatorname{Re}\left[\rho_{1}(\boldsymbol{r}) e^{-i \omega t}\right]+\rho_{2}(\boldsymbol{r}) .
\end{aligned}
$$

Here, subscript " 1 " denotes the first-order, complex-valued, time-harmonic acoustic fields $\propto \mathrm{Ma}^{1}$, and subscript "2" the second-order, real-valued, time-averaged steady fields $\propto \mathrm{Ma}^{2}$. We do not compute the oscillating second-order fields containing $e^{ \pm i 2 \omega t}$ as these have zero time average. In contrast, we do compute the oscillating first-order fields because products of these appear as source terms for the time-averaged second-order fields. In Eq. (2), real-valued, physical firstorder fields $\tilde{A}_{1}(\boldsymbol{r}, t)$ are written in terms of their complexvalued amplitude $A_{1}(\boldsymbol{r})$ as $\tilde{A}_{1}(\boldsymbol{r}, t)=\operatorname{Re}\left[A_{1}(\boldsymbol{r}) e^{-i \omega t}\right]$. The time average of a product of two real-valued, physical first-order fields $\tilde{A}_{1}(\boldsymbol{r}, t)$ and $\tilde{B}_{1}(\boldsymbol{r}, t)$ is then given in terms of the complex-valued amplitudes $A_{1}(\boldsymbol{r})$ and $B_{1}(\boldsymbol{r})$ as $\left\langle\tilde{A}_{1}(\boldsymbol{r}, t) \tilde{B}_{1}(\boldsymbol{r}, t)\right\rangle=\frac{1}{2} \operatorname{Re}\left[A_{1}(\boldsymbol{r}) B_{1}^{*}(\boldsymbol{r})\right]$.

\section{A. Pressure acoustics with boundary layers}

The complex-valued acoustic pressure $p_{1}(\boldsymbol{r})$ satisfies a Helmholtz equation with a complex-valued compressional wave number $k_{\mathrm{c}}$ having the real part $k_{0}=\frac{\omega}{c_{\mathrm{f}}}$. We apply the effective boundary condition for $p_{1}(\boldsymbol{r})$ recently derived by Bach and Bruus [28], which takes the viscous boundary layer into account at the domain boundary $\partial \Omega$. We introduce the inward normal derivative $\partial_{\perp}=-\boldsymbol{n} \cdot \boldsymbol{\nabla}, \boldsymbol{n}$ being the outwardpointing surface normal vector, and the effective inward normal displacement $U_{1 \perp}(\boldsymbol{r})$ of the wall in terms of the actual displacement $\boldsymbol{u}_{1}(\boldsymbol{r})$ :

$$
\begin{array}{cc}
\nabla^{2} p_{1}+k_{\mathrm{c}}^{2} p_{1}=0, \quad k_{\mathrm{c}}=\left(1+i \frac{\Gamma_{\mathrm{fl}}}{2}\right) k_{0}, & \boldsymbol{r} \in \Omega, \\
\partial_{\perp} p_{1}+\frac{i}{k_{\mathrm{s}}}\left(k_{\mathrm{c}}^{2} p_{1}+\partial_{\perp}^{2} p_{1}\right)=\frac{k_{0}^{2} U_{1 \perp}(\boldsymbol{r})}{\kappa_{\mathrm{fl}}}, & \boldsymbol{r} \in \partial \Omega, \\
U_{1 \perp}(\boldsymbol{r})=\frac{1}{1-i \Gamma_{\mathrm{fl}}}\left(-\boldsymbol{n}-\frac{i}{k_{\mathrm{s}}} \nabla\right) \cdot \boldsymbol{u}_{1}, & \boldsymbol{r} \in \partial \Omega .
\end{array}
$$

Here, $i=\sqrt{-1}, \Gamma_{\mathrm{fl}}$ is the weak bulk damping coefficient, and $k_{\mathrm{s}}$ is the viscous boundary layer wave number, given in terms of the viscous boundary-layer width $\delta$, the dynamic viscosity $\eta_{\mathrm{fl}}$, the bulk viscosity $\eta_{\mathrm{fl}}^{\mathrm{b}}$, and the isentropic compressibility $\kappa_{\mathrm{fl}}=\frac{1}{\rho_{\mathrm{fl}}}\left(\frac{\partial \rho}{\partial p}\right)_{S}=\frac{1}{\rho_{\mathrm{ff}} c_{\mathrm{fl}}^{2}}$ of the fluid,

$$
\Gamma_{\mathrm{fl}}=\left(\frac{4}{3}+\frac{\eta_{\mathrm{fl}}^{\mathrm{b}}}{\eta_{\mathrm{fl}}}\right) \eta_{\mathrm{fl}} \kappa_{\mathrm{fl}} \omega \ll 1, \quad k_{\mathrm{s}}=\frac{1+i}{\delta}, \quad \delta=\sqrt{\frac{2 \eta_{\mathrm{fl}}}{\rho_{\mathrm{fl}} \omega}} .
$$

Equation (3) for the acoustic pressure $p_{1}$ is valid for boundarylayer widths $\delta$ much smaller than both the acoustic length scale $k_{0}^{-1}$ and the geometry length cavity $L_{\text {geom }}$, a condition which is usually satisfied.

From the acoustic pressure $p_{1}$ we directly obtain the irrotational acoustic velocity $\boldsymbol{v}_{1}$ and the acoustic mass density $\rho_{1}$ in Eq. (2) through the relations [28]

$$
\begin{aligned}
& \boldsymbol{v}_{1}=-\frac{i\left(1-i \Gamma_{\mathrm{fl}}\right)}{\omega \rho_{\mathrm{fl}}} \nabla p_{1}, \quad \nabla \times \boldsymbol{v}_{1}=\mathbf{0}, \\
& \rho_{1}=\rho_{\mathrm{fl}} \kappa_{\mathrm{fl}} p_{1} .
\end{aligned}
$$

Further, we define the following relevant time-averaged firstorder products: the acoustic energy density $E_{\mathrm{ac}}$, kinetic energy density $E_{\text {kin }}$, potential energy density $E_{\text {pot }}$, energy flux density vector $S_{\mathrm{ac}}$, as well as the time-averaged acoustic angular 
momentum density $\mathcal{L}_{\mathrm{ac}}$ with respect to the unperturbed fluid position $\boldsymbol{r},{ }^{1}$

$$
\begin{array}{rlrl}
E_{\mathrm{ac}} & =E_{\mathrm{kin}}+E_{\mathrm{pot}}, & E_{\mathrm{kin}} & =\frac{1}{4} \rho_{\mathrm{ff}}\left|\boldsymbol{v}_{1}\right|^{2}, \\
\boldsymbol{S}_{\mathrm{ac}} & =\left\langle\tilde{p}_{1} \tilde{\boldsymbol{v}}_{1}\right\rangle, & E_{\mathrm{pot}} & =\frac{1}{4} \kappa_{\mathrm{fl}}\left|p_{1}\right|^{2}, \\
\mathcal{L}_{\mathrm{ac}} & =\left\langle\tilde{\boldsymbol{d}}_{1} \times\left(\rho_{\mathrm{fl}} \tilde{\boldsymbol{v}}_{1}\right)\right\rangle, & \boldsymbol{d}_{1}(\boldsymbol{r})=\frac{i}{\omega} \boldsymbol{v}_{1}(\boldsymbol{r}) .
\end{array}
$$

Here, $\boldsymbol{d}_{1}(\boldsymbol{r})$ is the fluid displacement at position $\boldsymbol{r}$.

\section{B. Acoustic streaming at fluid resonance}

Outside the narrow viscous boundary layers of width $\delta \lesssim$ $500 \mathrm{~nm}$ (for water at $\mathrm{MHz}$ frequencies), the second-order acoustic streaming velocity $\boldsymbol{v}_{2}$ is described as a Stokes flow driven by the acoustic body force $f_{\text {ac }}$ in the domain $\Omega$ [see Eq. (8) in Ref. [40] and Eq. (52b) in Ref. [28]], and by the slip velocity $\boldsymbol{v}_{2}^{\text {slip }}$ at the domain boundary $\partial \Omega$ [see Eq. (A19) in Ref. [28]]:

$$
\begin{aligned}
0 & =\nabla \cdot \boldsymbol{v}_{2}, \quad \boldsymbol{r} \in \Omega, \\
\mathbf{0} & =-\nabla p_{2}+\eta_{\mathrm{f}} \nabla^{2} \boldsymbol{v}_{2}+\boldsymbol{f}_{\mathrm{ac}}, \quad \boldsymbol{r} \in \Omega, \\
\boldsymbol{v}_{2} & =\boldsymbol{v}_{2}^{\text {slip }}, \quad \boldsymbol{r} \in \partial \Omega .
\end{aligned}
$$

Here, $\boldsymbol{f}_{\mathrm{ac}}$ in $\Omega$ and $\boldsymbol{v}_{2}^{\text {slip }}$ at $\partial \Omega$, are given by

$$
\begin{aligned}
\boldsymbol{f}_{\mathrm{ac}} & =\frac{\Gamma_{\mathrm{fl}} \omega}{c_{\mathrm{fl}}^{2}} \boldsymbol{S}_{\mathrm{ac}}, \\
\boldsymbol{v}_{2}^{\text {slip }} & =\frac{1}{2} \kappa_{\mathrm{ff}} \boldsymbol{S}_{\mathrm{ac}}-\frac{1}{2 \rho_{\mathrm{fl}} \omega} \nabla_{\|}\left[E_{\mathrm{kin}}-2 E_{\mathrm{pot}}\right]+\frac{3}{4 \omega} \operatorname{Re}\left[i \partial_{\perp} v_{1 \perp} \boldsymbol{v}_{1 \|}^{*}\right],
\end{aligned}
$$

where we have used the expression for the slip velocity $\boldsymbol{v}_{2}^{\text {slip }}$ valid near a fluid resonance, in which case the magnitude $\left|\boldsymbol{v}_{1}\right|$ of the acoustic velocity $\boldsymbol{v}_{1}$ in the bulk is much larger than that of the wall velocity $\left|\omega \boldsymbol{u}_{1}\right| \ll\left|\boldsymbol{v}_{1}\right|$ [see Eq. (55) in Ref. [28] for the slip velocity including the wall motion and Stokes drift]. We use the name bulk-driven acoustic streaming for the streaming driven by the acoustic body force $f_{\text {ac }}$ in Eq. (7d), and the name boundary-driven streaming for the streaming driven by the slip velocity $\boldsymbol{v}_{2}^{\text {slip }}$ in Eq. (7e). Note that $\boldsymbol{f}_{\text {ac }}$ increases with the frequency squared, as $\Gamma_{\mathrm{fl}} \propto \omega$, whereas $\boldsymbol{v}_{2}^{\text {slip }}$ is independent of the frequency, as $\nabla_{\|}, \partial_{\perp} \sim k_{0}=\frac{1}{c_{\sharp}} \omega$.

One central quantity in the governing equations (7) for the acoustic streaming is the acoustic energy flux density $\boldsymbol{S}_{\mathrm{ac}}$, which enters both in the body force $\boldsymbol{f}_{\text {ac }}$ [Eq. (7d)] and in the slip velocity $\boldsymbol{v}_{2}^{\text {slip }}$ [Eq. (7e)]. This quantity is rotating (has

\footnotetext{
${ }^{1}$ The standard definition of the full angular momentum density is $\mathcal{L}=\boldsymbol{r}^{\mathrm{L}}[t] \times\left\{\rho\left[\boldsymbol{r}^{\mathrm{L}}(t), t\right] \boldsymbol{v}\left[\boldsymbol{r}^{\mathrm{L}}(t), t\right]\right\}$, defined in terms of the instantaneous Lagrangian fluid position $\boldsymbol{r}^{\mathrm{L}}(t)=\boldsymbol{r}+\tilde{\boldsymbol{d}}_{1}(\boldsymbol{r}, t)+\mathcal{O}\left(\mathrm{Ma}^{2}\right)$, where $\boldsymbol{r}$ is the equilibrium position of the fluid. Taylor expanding $\rho\left[\boldsymbol{r}^{\mathrm{L}}(t), t\right] \approx \rho(\boldsymbol{r}, t)+\tilde{\boldsymbol{d}}_{1} \cdot \nabla \rho$ and $\boldsymbol{v}\left[\boldsymbol{r}^{\mathrm{L}}(t), t\right] \approx \boldsymbol{v}(\boldsymbol{r}, t)+\tilde{\boldsymbol{d}}_{1}$. $\nabla v(r, t)$, and using the expansion in Eq. (2) we obtain the timeaveraged full angular momentum density $\mathcal{L}=\boldsymbol{r} \times\left\{\rho_{\mathrm{f}}\left[\boldsymbol{v}_{2}+\left\langle\tilde{\boldsymbol{d}}_{1}\right.\right.\right.$. $\left.\left.\left.\nabla \tilde{\boldsymbol{v}}_{1}\right\rangle\right]+\left\langle\tilde{\rho}_{1} \tilde{\boldsymbol{v}}_{1}\right\rangle\right\}+\left\langle\tilde{\boldsymbol{d}}_{1} \times\left(\rho_{\mathrm{ff}} \tilde{\boldsymbol{v}}_{1}\right)\right\rangle$. Here, the last term is $\mathcal{L}_{\text {ac }}$ from Eq. (6c) and expresses the angular momentum density of each fluid particle around its equilibrium position $\boldsymbol{r}$.
}

a nonzero curl) if the acoustic fields are rotating, as seen by taking the curl of $\boldsymbol{S}_{\mathrm{ac}}=\left\langle\tilde{p}_{1} \tilde{\boldsymbol{v}}_{1}\right\rangle$ and using Eq. (5a) with $\boldsymbol{\nabla} p_{1} \approx i \omega \rho_{\mathrm{fl}} \boldsymbol{v}_{1}$ and $\boldsymbol{\nabla} \times \boldsymbol{v}_{1}=\mathbf{0}$,

$$
\nabla \times S_{\mathrm{ac}}=\omega^{2} \mathcal{L}_{\mathrm{ac}}
$$

where $\mathcal{L}_{\text {ac }}$ is the time-averaged acoustic angular momentum density defined in Eq. (6c). Using Stokes theorem for any closed loop shows that the energy flux density $\boldsymbol{S}_{\mathrm{ac}}$, and therefore the body force $f_{\text {ac }}$, points in a direction which rotates around areas with high acoustic angular momentum density

$$
\oint \boldsymbol{f}_{\mathrm{ac}} \cdot d \boldsymbol{l}=\frac{\Gamma_{\mathrm{f}} \omega}{c_{\mathrm{fl}}^{2}} \oint \boldsymbol{S}_{\mathrm{ac}} \cdot d \boldsymbol{l}=\frac{\Gamma_{\mathrm{ff}} \omega^{3}}{c_{\mathrm{fl}}^{2}} \int \mathcal{L}_{\mathrm{ac}} \cdot \boldsymbol{n} d A .
$$

Hence, bulk-driven streaming is a consequence of acoustic fields with a nonzero acoustic angular momentum density $\mathcal{L}_{\mathrm{ac}}$ defined in Eq. (6c). We refer to acoustic fields with nonzero $\mathcal{L}_{\text {ac }}$ as "rotating acoustics."

\section{ACOUSTIC RESONANCE MODES IN A RECTANGULAR BOX WITH VISCOUS BOUNDARY LAYERS}

We consider the rectangular-box-shaped cavity of dimensions $L_{x} \times L_{y} \times L_{z}$, where $0 \leqslant x \leqslant L_{x}, 0 \leqslant y \leqslant L_{y}$, and $0 \leqslant$ $z \leqslant L_{z}$. Below, we give the solutions for the resonance modes of the acoustic pressure $p_{1}$ satisfying Eq. (3) in this geometry with viscous boundary layers. We refer the reader to Appendix A for the detailed derivations. The resulting expressions are valid for frequencies close to resonances of a high $Q$ factor $Q \gg 1$ and for narrow boundary layers [see Eq. (A3)]. We then evaluate the second-order quantities $\boldsymbol{S}_{\mathrm{ac}}$, $\boldsymbol{f}_{\mathrm{ac}}$, and $\mathcal{L}_{\text {ac }}$ which vanish for all single-mode resonances but not for double-mode resonances, where two single modes are excited simultaneously by the same frequency.

\section{A. Single-mode resonances}

First, neglecting viscosity, the resonance solution for $p_{1}$ is proportional to the eigenfunctions $R^{l m n}(\boldsymbol{r})$ of the Helmholtz equation (3a), which are the usual hard-wall standing resonance modes with integer number $l, m$, and $n$ half-waves in the $x, y$, and $z$ directions, respectively,

$$
p_{1}^{l m n} \propto R^{l m n}(\boldsymbol{r})=\cos \left(K_{x}^{l} x\right) \cos \left(K_{y}^{m} y\right) \cos \left(K_{z}^{n} z\right) .
$$

Here, the wave numbers $K_{x}^{l}, K_{y}^{m}$, and $K_{z}^{n}$ are real-valued, purely geometrical quantities that dictate the resonance wave numbers $K_{0}^{l m n}$ :

$$
\begin{aligned}
K_{x}^{l} & =\frac{l \pi}{L_{x}}, \quad K_{y}^{m}=\frac{m \pi}{L_{y}}, \quad K_{z}^{n}=\frac{n \pi}{L_{z}}, \\
K_{0}^{l m n} & =\sqrt{\left(K_{x}^{l}\right)^{2}+\left(K_{y}^{m}\right)^{2}+\left(K_{z}^{n}\right)^{2}} .
\end{aligned}
$$

In the presence of viscosity and hence viscous boundary layers, the resonant wave numbers $k_{0}^{l m n}$ and angular frequencies $\omega^{l m n}$ are down-shifted slightly relative to the inviscid values 


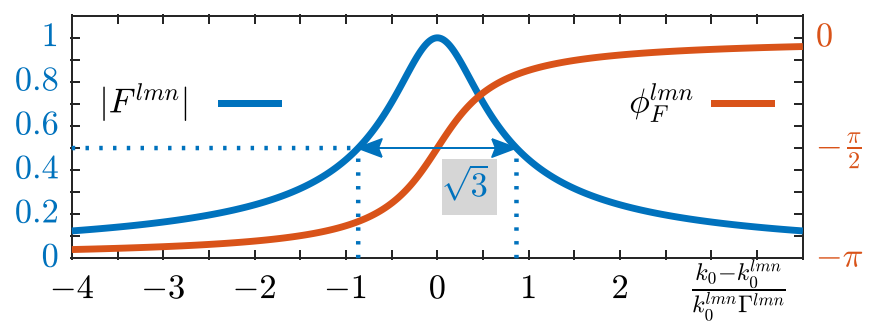

FIG. 1. The modulus $\left|F^{l m n}\right|$ (left axis) and phase $\phi_{F}^{\text {lmn }}$ (right axis) of the frequency-dependent factor $F^{l m n}$ in Eq. (12c). The double arrow marks the full-width-at-half-maximum linewidth $\sqrt{3} k_{0}^{l m n} \Gamma^{l m n}$ of $\left|F^{l m n}\right|$.

due to the boundary-layer damping coefficients $\Gamma_{\mathrm{bl}}^{l m n}$ :

$$
\begin{aligned}
k_{0}^{l m n}= & \left(1-\frac{1}{2} \Gamma_{\mathrm{bl}}^{l m n}\right) K_{0}^{l m n}, \quad \omega^{l m n}=k_{0}^{l m n} c_{\mathrm{fl}} \\
\Gamma_{\mathrm{bl}}^{l m n}= & \left(\frac{K_{x}^{l}}{K_{0}^{l m n}}\right)^{2}\left(\frac{\delta}{L_{y}^{m}}+\frac{\delta}{L_{z}^{n}}\right)+\left(\frac{K_{y}^{m}}{K_{0}^{l m n}}\right)^{2}\left(\frac{\delta}{L_{z}^{n}}+\frac{\delta}{L_{x}^{l}}\right) \\
& +\left(\frac{K_{z}^{n}}{K_{0}^{l m n}}\right)^{2}\left(\frac{\delta}{L_{x}^{l}}+\frac{\delta}{L_{y}^{m}}\right)
\end{aligned}
$$

where $L_{x}^{l}=\int_{0}^{L_{x}} \cos ^{2}\left(K_{x}^{l} x\right) d x=\frac{1}{2}\left(1+\delta_{0 l}\right) L_{x}$ and similarly for $L_{y}^{m}$ and $L_{z}^{n}$. As an example, a pure $x$ mode will have $K_{0}^{l 00}=$ $K_{x}^{l}$ and $K_{y}^{0}=K_{z}^{0}=0$ giving $\Gamma_{\mathrm{bl}}^{l 00}=\frac{\delta}{L_{y}}+\frac{\delta}{L_{z}}$ corresponding to the boundary-layer damping from the four boundaries parallel to the $x$ axis. The total damping coefficient $\Gamma^{l m n}$ of the resonance mode $l m n$ includes both the boundary-layer-damping coefficients $\Gamma_{\mathrm{bl}}^{l m n}$ and the bulk-damping coefficient $\Gamma_{\mathrm{fl}}^{l m n}$, given in Eq. (4) with $\omega=\omega^{l m n}$,

$$
\Gamma^{l m n}=\Gamma_{\mathrm{bl}}^{l m n}+\Gamma_{\mathrm{fl}}^{l m n} .
$$

For frequencies close to the resonance frequencies, we have $k_{0} \approx k_{0}^{l m n}$ or $\omega \approx \omega^{l m n}$, and the resonance pressure mode $p_{1}^{l m n}$ satisfying Eq. (3) is the product of a complex-valued amplitude $P_{1}^{l m n}$, an internal frequency dependency $F^{l m n}\left(k_{0}\right)$, and the usual real-valued, hard-wall spatial dependency $R^{l m n}(\boldsymbol{r})$ from Eq. (9a):

$$
\begin{aligned}
p_{1}^{l m n}\left(k_{0}, \boldsymbol{r}\right) & =P_{1}^{l m n} F^{l m n}\left(k_{0}\right) R^{l m n}(\boldsymbol{r}) \text { for } k_{0} \approx k_{0}^{l m n} \\
P_{1}^{l m n} & =\frac{\rho_{\mathrm{fl}} c_{\mathrm{fl}}^{2}}{\Gamma^{l m n}} \frac{\int_{\partial \Omega} U_{1 \perp} R^{l m n} d A}{\int_{\Omega}\left(R^{l m n}\right)^{2} d V}=\left|P_{1}^{l m n}\right| e^{i \phi_{\mathrm{act}}^{l m n}}, \\
F^{l m n}\left(k_{0}\right) & =\frac{\frac{1}{2} k_{0}^{l m n} \Gamma^{l m n}}{\left(k_{0}-k_{0}^{l m n}\right)+i \frac{1}{2} k_{0}^{l m n} \Gamma^{l m n}}=\left|F^{l m n}\right| e^{i \phi_{F}^{l m n}}
\end{aligned}
$$

Here, we have also given $P_{1}^{l m n}$ and $F^{l m n}$ in their polar form and introduced the corresponding external actuation phase $\phi_{\text {act }}^{l m n}$ and internal frequency-dependent phase $\phi_{F}^{l m n}$. The phase $\phi_{F}^{l m}$ is plotted in Fig. 1 together with the modulus $\left|F^{l m n}\right|$. The total phase $\phi_{\text {tot }}^{l m n}$ of the mode $\operatorname{lm} n$ is the sum of these phases:

$$
\phi_{\mathrm{tot}}^{l m n}=\phi_{\mathrm{act}}^{l m n}+\phi_{F}^{l m n}
$$

\section{B. Double-mode resonances}

The bulk-driven acoustic streaming is driven by the body force $f_{\text {ac }}$, defined in Eq. (7d) and restated here solely in terms of the pressure $p_{1}$, by using $\boldsymbol{v}_{1} \approx \frac{-i}{\omega \rho_{\mathrm{fl}}} \nabla p_{1}$ as given in Eq. (5a):

$$
\boldsymbol{f}_{\mathrm{ac}}=\frac{\Gamma_{\mathrm{fl}} \omega}{c_{\mathrm{fl}}^{2}}\left\langle\tilde{p}_{1} \tilde{\boldsymbol{v}}_{1}\right\rangle=\Gamma_{\mathrm{fl}} \kappa_{\mathrm{fl}} \frac{1}{2} \operatorname{Re}\left[i p_{1} \nabla p_{1}^{*}\right] .
$$

For any single-mode resonance $l m n$ of the form of Eq. (12a), this expression will vanish since $i p_{1}^{l m n}$ is exactly $\frac{\pi}{2}$ out of phase with $\nabla p_{1}^{l m n}$. This motivates us to consider double-mode resonances, which occur if two single modes $l m n$ and $l ' m n^{\prime}$ overlap, such that they can be excited simultaneously at the same frequency $f=\frac{1}{2 \pi} k_{0} c_{\mathrm{fl}}$, with $k_{0}^{l m n} \approx k_{0} \approx k_{0}^{l m i n}$. In that case, the total pressure is the sum of two modes

$$
p_{1}=p_{l^{\prime} m n}^{l m n}=p_{1}^{l m n}+p_{1}^{l^{\prime \prime m} n^{\prime}}
$$

and the body force $\boldsymbol{f}_{\mathrm{ac}}$ in Eq. (14) becomes $\boldsymbol{f}_{l^{\prime m} n n}^{l m n}$ :

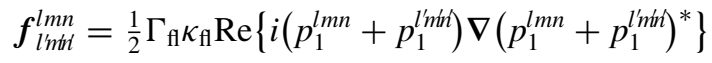

$$
\begin{aligned}
& =\frac{1}{2} \Gamma_{\mathrm{fl}} \kappa_{\mathrm{fl}}\left\{\operatorname{Re}\left[i p_{1}^{l m n} \nabla\left(p_{1}^{l^{\prime} m i n}\right)^{*}\right]-\operatorname{Re}\left[p_{1}^{l^{\prime \prime m} i n} \nabla\left(i p_{1}^{l m n}\right)^{*}\right]\right\} .
\end{aligned}
$$

Inserting the resonance mode Eq. (12a) with $P_{1}^{l m n}$ in polar form yields

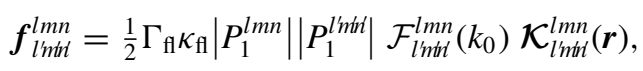

$$
\begin{aligned}
& \mathcal{F}_{l m i n}^{l m n}\left(k_{0}\right)=\operatorname{Re}\left\{i F^{l m n} e^{i \phi_{\mathrm{act}}^{l m n}}\left(F^{l^{\prime} m i n} e^{i \phi_{\mathrm{act}}^{l i m i}}\right)^{*}\right\}, \\
& \mathcal{K}_{l^{\prime \prime m} n}^{l m n}(\boldsymbol{r})=R^{l m n} \nabla R^{l^{\prime m i n}}-R^{l^{\prime \prime m} \dot{ }} \nabla R^{l m n} .
\end{aligned}
$$

Here, $\mathcal{F}_{l \text { min }}^{l m n}\left(k_{0}\right)$ gives the essential frequency dependency (as $\Gamma_{\mathrm{fl}}$ is nearly constant over the width of the double mode), while $\mathcal{K}_{l^{\prime} \text { min }}^{\operatorname{lmn}}(\boldsymbol{r})$ with the dimension of a wave vector gives the spatial dependency and direction of the double-mode body force. Furthermore, we obtain the double-mode energy flux density $\boldsymbol{S}_{l m n}^{l^{\prime} m h^{\prime}}$ and acoustic angular momentum density $\mathcal{L}_{l^{\prime \prime m} n}^{l m n}$ directly from Eq. (17a) by use of Eqs. (7d) and (8a):

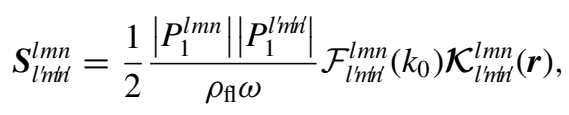

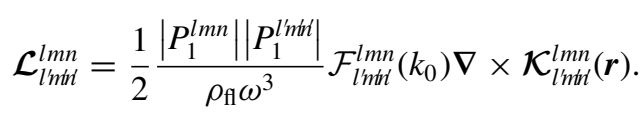

Note that $\mathcal{F}_{l^{l m} \text { in }}^{\operatorname{lm}}\left(k_{0}\right)$ is normalized to be between -1 and 1 , whereas $\mathcal{K}_{l^{\prime \prime m n} n}^{l m n}(\boldsymbol{r})$ and $\nabla \times \mathcal{K}_{l^{\prime m} n \dot{ }}^{l m n}(\boldsymbol{r})=2 \nabla R^{l m n} \times \nabla R^{l^{\prime \prime m i n}}$ has the maximum amplitudes $k_{0}$ and $2 k_{0}^{2}$, respectively.

In Fig. 2 we show results for the nine lowest-frequency double modes $l m 0+m l 0$ in a square cavity. We plot the body force $\boldsymbol{f}_{m l 0}^{l m 0} \propto \mathcal{K}_{m l 0}^{l m 0}$ and the $z$ component of the acoustic angular momentum $\mathcal{L}_{m l 0}^{\operatorname{lm} 0} \propto \nabla \times \mathcal{K}_{m l 0}^{l m 0}$. In the Supplemental Material [41] we show 55 examples of double-mode resonances $l m 0+l^{\prime} m 0$ with $l, m, l^{\prime}, m^{\prime}=0,1,2,3,4$ in rectangular 

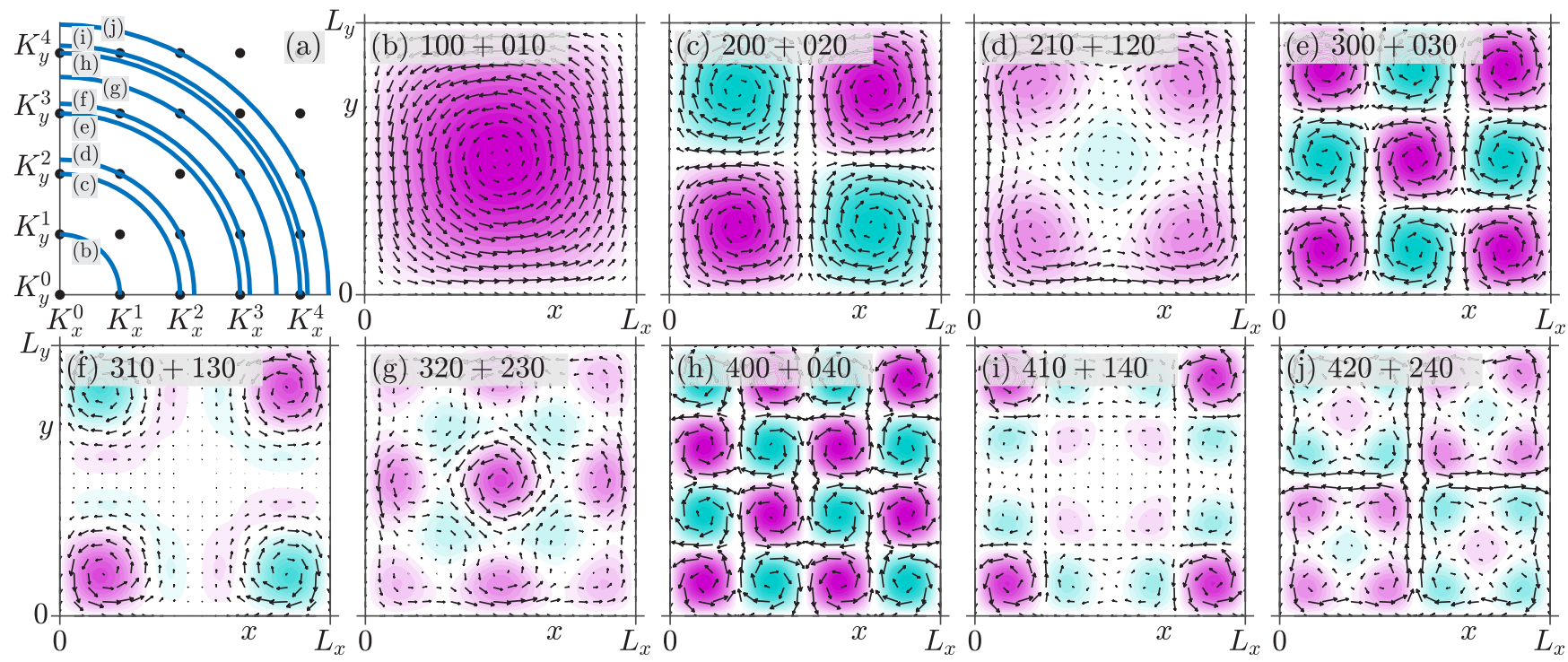

FIG. 2. Rotating acoustics in the $x y$ plane $\left(K_{z}^{n}=0\right)$ of a square cavity with $L_{x}=L_{y}$. (a) $K_{x}-K_{y}$ space diagram showing the allowed values of $K_{x}^{l}$ and $K_{y}^{m}$ (black dots). Each quarter circle (b)-(j) corresponds to the angular frequency $c_{\mathrm{fl}} \sqrt{\left(K_{x}^{l}\right)^{2}+\left(K_{y}^{m}\right)^{2}}$, where the double mode $\operatorname{lm} 0+m l 0$ is excited. (b)-(j) The corresponding acoustic rotation of the double mode $\operatorname{lm} 0+m l 0$, where the arrows represent the body force $f_{m l 0}^{l m 0}$ and the colors show the $z$ component of the spatial dependency $\nabla \times \mathcal{K}_{m l 0}^{l m 0}$ in Eq. (18b) of the acoustic angular momentum density from $-2 k_{0}^{2}$ (light cyan) to $2 k_{0}^{2}$ (dark magenta).

cavities with aspect ratios between 1 and 4 , and we provide a MATLAB code to compute these modes.

\section{CONDITIONS FOR OBTAINING A STRONG ACOUSTIC BODY FORCE}

The magnitude of the body force $\boldsymbol{f}_{l m h t}^{l m n}$, the acoustic energy flux density $\boldsymbol{S}_{l h n t i}^{l m n}$, and the acoustic angular momentum density $\mathcal{L}_{l \text { lmit }}^{\operatorname{lmn}}$ are all proportional to the frequency-dependent factor $\mathcal{F}_{l m i n}^{\text {Imn }}\left(k_{0}\right)$ in Eq. (17b). To study this factor, we rewrite it to emphasize the total phase difference $\phi_{\mathrm{tot}}^{l i n i t}-\phi_{\mathrm{tot}}^{l m n}$ between the single-mode phases given in Eq. (13):

$$
\mathcal{F}_{l m h i}^{l m n}\left(k_{0}\right)=\left|F^{l m n}\right|\left|F^{l i n h i}\right| \sin \left(\phi_{\mathrm{tot}}^{l m i n}-\phi_{\mathrm{tot}}^{l m n}\right) .
$$

Consequently, two conditions must be fulfilled to yield a strong body force: (i) the overlap condition, i.e., the two single-mode resonances must have a large overlap in frequency space to ensure a large value of the amplitude $\left|F^{l m n}\right|\left|F^{l m h i}\right|$, and (ii) the phase condition, i.e., the difference $\phi_{\mathrm{tot}}^{l \operatorname{mit}}-\phi_{\mathrm{tot}}^{l m n}$ in phases must be close to $\pm \frac{\pi}{2}$ to ensure a large value of the sine factor.

\section{A. Overlap condition: Aspect ratios}

The condition that both $\left|F^{l m n}\right|$ and $\left|F^{l i n i n}\right|$ are large in Eq. (19) is satisfied if the modes overlap. This occurs if the resonance frequencies are nearly identical, $k_{0}^{l m n} \approx k_{0}^{l \text { lmhit }}$ or, equivalently,

$$
\left(\frac{l \pi}{L_{x}}\right)^{2}+\left(\frac{m \pi}{L_{y}}\right)^{2}+\left(\frac{n \pi}{L_{z}}\right)^{2} \approx\left(\frac{l^{\prime} \pi}{L_{x}}\right)^{2}+\left(\frac{m^{\prime} \pi}{L_{y}}\right)^{2}+\left(\frac{n^{\prime} \pi}{L_{z}}\right)^{2},
$$

where we have used that $\Gamma_{\mathrm{bl}}^{l m n} \ll 1$ in Eq. (10a) so $k_{0}^{l m n} \approx$ $K_{0}^{l m n}$. In the general case, Eq. (20) gives a myriad of possible mode combinations. If we restrict ourselves to horizontal modes, where $n=n^{\prime}=0$, we can solve for the aspect ratio $A=\frac{L_{x}}{L_{y}}$ in Eq. (20):

$$
A=\frac{L_{x}}{L_{y}} \approx \sqrt{\frac{l^{2}-l^{2}}{m^{2}-m^{2}}} .
$$

Listing the integers $l, m, l^{\prime}, m^{\prime}$ for which $A$ is real, we find that the square cavity with $A \approx 1$ is the richest case having the highest number of allowed double-mode excitations. In Fig. 2, we show the first nine horizontal double-mode excitations in a square cavity with $A=1$ and $n=n^{\prime}=0$. In this case, the condition in Eq. (20) corresponds to two points $\left(\frac{l \pi}{L_{x}}, \frac{m \pi}{L_{y}}\right)$ and $\left(\frac{l i \pi}{L_{x}}, \frac{m \pi}{L_{y}}\right)$ both lying on the same quarter circle of radius $K_{0}$, as illustrated by the black dots in Fig. 2(a).

\section{B. Phase condition: Rotating versus nonrotating actuation}

The straightforward way to obtain rotating acoustics is to actuate a given system with two transducers running with a phase difference of $\phi_{\mathrm{act}}^{l \text { hnt }}-\phi_{\mathrm{act}}^{l m n}= \pm \frac{\pi}{2}$, a technique that has been used in many experiments to generate acoustic radiation torques [26,31-35]. Remarkably, even in the standard case of microfluidic ultrasound experiments driven by a single piezoelectric transducer with a nonrotating actuation, $\phi_{\text {act }}^{l^{\prime n h i l}-}$ $\phi_{\mathrm{act}}^{l m n}=0$, a rotating acoustic field may nevertheless be generated because the total phase difference $\phi_{\text {tot }}^{l i n h i}-\phi_{\text {tot }}^{l m n}=\phi_{F}^{l^{\prime \prime h h i}}-$ $\phi_{F}^{l m n}$ may be close to $\pm \frac{\pi}{2}$ due to the internal phases $\phi_{F}^{l m n}$ of the frequency-dependent factors $F^{l m n}\left(k_{0}\right)$ [see Eq. (12c) and Fig. 1]. 


\section{NONROTATING ACTUATION OF ROTATING DOUBLE-MODE RESONANCES IN A NEARLY SQUARE CAVITY}

We now show how a nonrotating actuation can lead to a rotating double-mode resonance in a nearly square cavity characterized by the average side length $L^{\star}=\frac{1}{2}\left(L_{x}+L_{y}\right)$ and the small symmetry breaking $d=L_{x}-L_{y}$ :

$$
L_{x}=L^{\star}+\frac{1}{2} d, \quad L_{y}=L^{\star}-\frac{1}{2} d, \quad \text { with }|d| \ll L^{\star} .
$$

The magnitude of the resulting acoustic body force $\boldsymbol{f}_{\text {ac }}$ depends strongly on the symmetry breaking $d$, and for a given double mode we determine how much the aspect ratio $A=$ $L_{x} / L_{y} \approx 1+d / L^{\star}$ should deviate from unity to maximize $\boldsymbol{f}_{\mathrm{ac}}$. Specifically, we consider the symmetric double-mode excitations $\ln 0+m l 0$ in the horizontal $x y$ plane of the type shown in Fig. 2. For such double modes, the analysis simplifies significantly, not only because $z$ drops out, but also because the damping coefficients $\Gamma^{l m 0}$ and $\Gamma^{m l 0}$ from Eqs. (10b) and (11) are almost identical. In short, we consider the following situation:

$$
\begin{array}{ll}
\text { Nearly square cavity: } & L_{x} \approx L^{\star} \approx L_{y}, \\
\text { Horizontal } x y \text { modes: } & n=n^{\prime}=0, \\
\text { Symmetric double modes: } & l^{\prime} m^{\prime}=m l, \\
\text { Nonrotating actuation: } & \phi_{\mathrm{act}}^{l m 0}=\phi_{\mathrm{act}}^{m l 0}, \\
\text { Similar damping coefficients: } & \Gamma^{l m 0} \approx \Gamma^{m l 0} .
\end{array}
$$

To analyze the strength of the acoustic body force, we examine the factor $\mathcal{F}_{l m 0}^{m l 0}\left(k_{0}\right)$ in Eq. (17b) that describes the frequency dependency of the acoustic body force. For the double mode $l m 0+m l 0$, we introduce the short two-subscript notation $\mathcal{F}_{l m}\left(k_{0}\right)$ for this quantity:

$$
\begin{aligned}
\mathcal{F}_{l m}\left(k_{0}\right) & =\mathcal{F}_{m l 0}^{l m 0}\left(k_{0}\right)=\operatorname{Re}\left\{i\left[F^{l m 0}\right]\left[F^{m l 0}\right]^{*}\right\} \\
& =\left|F^{l m 0}\right|\left|F^{m l 0}\right| \sin \left(\phi_{F}^{m l 0}-\phi_{F}^{l m 0}\right),
\end{aligned}
$$

where the functions $F^{l m 0}\left(k_{0}\right)$ and $F^{m l 0}\left(k_{0}\right)$ behave as shown in Fig. 1. Note that if the modes $\operatorname{lm} 0$ and $m l 0$ have equal resonance frequencies, they have full mode overlap, and the product $\left|F^{l m 0}\right|\left|F^{m l 0}\right|$ is maximized, while $\sin \left(\phi_{F}^{m l 0}-\phi_{F}^{l m 0}\right)$ vanishes, and there is no body force. To maximize $\mathcal{F}_{l m}\left(k_{0}\right)$, the two modes must therefore be separated sufficiently to ensure that the phase difference $\phi_{F}^{m l 0}-\phi_{F}^{l m 0}$ deviates from zero, but not too much, as a decreased mode overlap reduces the modulus product $\left|F^{l m 0}\right|\left|F^{m l 0}\right|$. Therefore, we cannot in this case perfectly fulfill both the overlap condition, Sec. IV A, and the phase condition, Sec. IV B, so the strongest body force is found at a certain mode separation $\Delta_{l m}=k_{0}^{l m 0}-k_{0}^{m l 0}$ between the resonance wave numbers, which gives only partial mode overlap and a phase difference that deviates from the ideal $\pm \frac{\pi}{2}$.

The mode separation $\Delta_{l m}$ is induced by the symmetry breaking $d$, and similar to Eq. (22), we introduce the center wave number $k_{l m}^{\star}=\frac{1}{2}\left(k_{0}^{l m 0}+k_{0}^{m l 0}\right)$ corresponding to the
TABLE I. Overview of the damping coefficients $\Gamma$, the fullwidth-at-half-maximum (FWHM) linewidths, and the mode separation $\Delta$.

\begin{tabular}{lcc}
\hline \hline Parameter & Symbol & Reference \\
\hline Bulk damping & $\Gamma_{\mathrm{fl}}$ & Eq. (4) \\
Boundary-layer damping & $\Gamma_{\mathrm{bl}}^{l m n}$ & Eq. (10b) \\
Total damping & $\Gamma^{l m n}$ & Eq. (11) \\
FWHM of $\left|F^{l m n}\right|$ & $\sqrt{3} k_{0}^{l m n} \Gamma^{l m n}$ & Fig. 1 \\
FWHM of $E_{\mathrm{ac}}^{l m n} \propto\left|F^{l m n}\right|^{2}$ & $k_{0}^{l m n} \Gamma^{l m n}$ & Eqs. (6), (12) \\
Optimal mode separation $\Delta_{l m}^{\mathrm{opt}}$ & $\frac{1}{\sqrt{3}} k_{l m}^{\star} \Gamma_{l m}^{\star}$ & Eq. (29b) \\
\hline \hline
\end{tabular}

center frequency between the two modes and write

$$
\begin{aligned}
k_{0}^{l m 0} & =k_{l m}^{\star}+\frac{1}{2} \Delta_{l m}, \quad k_{0}^{m l 0}=k_{l m}^{\star}-\frac{1}{2} \Delta_{l m}, \\
k_{l m}^{\star} & \approx\left(1-\frac{1}{2} \Gamma_{\mathrm{bl}}^{l m 0}\right) \sqrt{l^{2}+m^{2}} \frac{\pi}{L^{\star}} .
\end{aligned}
$$

The relation between the mode separation $\Delta_{l m}$, the symmetry breaking $d$, and the aspect ratio $A=L_{x} / L_{y}$ is found by inserting Eq. (22) into $k_{0}^{l m 0}=\sqrt{\left(\frac{l \pi}{L_{x}}\right)^{2}+\left(\frac{m \pi}{L_{y}}\right)^{2}}+\mathcal{O}\left(\Gamma^{l m 0}\right)$, expanding in the small ratio $\frac{d}{L^{\star}}$, and comparing with Eq. (25a),

$$
\Delta_{l m} \approx \frac{m^{2}-l^{2}}{\sqrt{m^{2}+l^{2}}} \frac{\pi}{L^{\star}} \frac{d}{L^{\star}} \approx \frac{m^{2}-l^{2}}{\sqrt{m^{2}+l^{2}}} \frac{\pi}{L^{\star}}(A-1) .
$$

Since the damping coefficients are nearly identical $\Gamma^{\operatorname{lm} 0} \approx$ $\Gamma_{l m}^{\star} \approx \Gamma^{m l 0}$, with $\Gamma_{l m}^{\star}=\frac{1}{2}\left(\Gamma^{l m 0}+\Gamma^{m l 0}\right)$ being the average value, it is convenient to scale all wave-number quantities by their common linewidth $\Gamma_{l m}^{\star} k_{l m}^{\star}$ (see the overview listed in Table I):

$$
\tilde{k}_{0}=\frac{k_{0}}{k_{l m}^{\star} \Gamma_{l m}^{\star}}, \quad \tilde{k}_{l m}^{\star}=\frac{1}{\Gamma_{l m}^{\star}}, \quad \tilde{\Delta}_{l m}=\frac{\Delta_{l m}}{k_{l m}^{\star} \Gamma_{l m}^{\star}} .
$$

In terms of these quantities, we write the frequency dependencies $F^{l m 0}\left(k_{0}\right)$ and $F^{m l 0}\left(k_{0}\right)$ from Eq. (12c) as

$$
\begin{aligned}
& F^{l m 0}\left(k_{0}\right)=\frac{1}{2\left(\tilde{k}_{0}-\tilde{k}_{l m}^{\star}\right)-\tilde{\Delta}_{l m}+i}, \\
& F^{m l 0}\left(k_{0}\right)=\frac{1}{2\left(\tilde{k}_{0}-\tilde{k}_{l m}^{\star}\right)+\tilde{\Delta}_{l m}+i} .
\end{aligned}
$$

In Figs. 3(a) and 3(b) we show as in Fig. 1 the modulus and phase, respectively, of $F^{\operatorname{lm} 0}$ and $F^{m l 0}$ for four different mode separations $\tilde{\Delta}_{l m}=0.10,0.58,1.50,3.00$. In Fig. 3(c) we show for the same values of $\tilde{\Delta}_{l m}$ the frequency dependency $\mathcal{F}_{l m}\left(k_{0}\right)$ from Eq. (24) of the acoustic body force. We note that there exists an optimal mode separation $\tilde{\Delta}_{l m}^{\text {opt }}$ that yields the largest possible value $\mathcal{F}_{l m}^{\text {opt }}$ of $\mathcal{F}_{l m}$ found at the optimal wave number $k_{0}^{\text {opt }}$. In Eq. (B5) of Appendix B we show that

$$
\begin{aligned}
& k_{0}^{\mathrm{opt}}=k_{l m}^{\star} \\
& \tilde{\Delta}_{l m}^{\mathrm{opt}}= \pm \frac{1}{\sqrt{3}}= \pm 0.58 \\
& \mathcal{F}_{l m}^{\mathrm{opt}}= \pm \frac{3 \sqrt{3}}{8}= \pm 0.65
\end{aligned}
$$



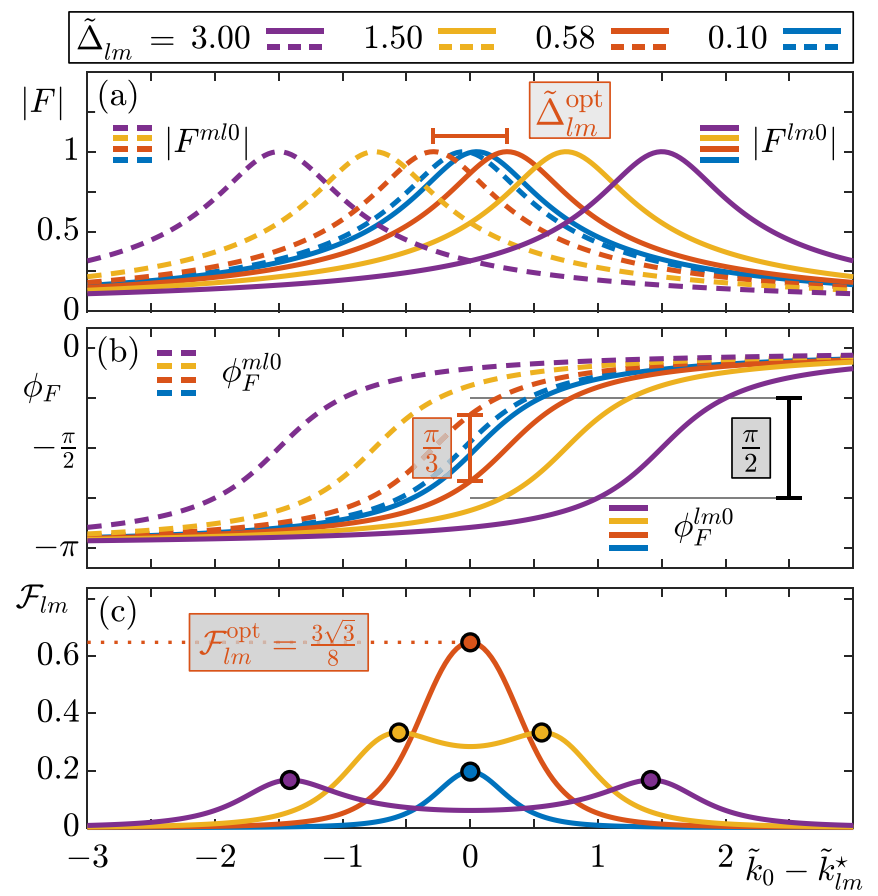

FIG. 3. Plots of $\left|F^{m l 0}\right|,\left|F^{l m 0}\right|, \phi_{F}^{m l 0}, \phi_{F}^{l m 0}$, and $\mathcal{F}_{l m}$ as a function of $\tilde{k}_{0}-\tilde{k}_{l m}^{\star}$, each for the four values $3.00,1.50,0.58$, and 0.10 of the mode separation $\tilde{\Delta}_{l m}$. (a) The modulus $|F|$ and (b) the phase $\phi_{F}$ of $F^{l m 0}$ (right, solid) and $F^{m l 0}$ (left, dashed) given in Eq. (28). At the optimal mode separation $\tilde{\Delta}_{l m}^{\text {opt }}=\frac{1}{\sqrt{3}} \approx 0.58$ (orange), the phase difference at the center frequency is $\frac{\pi}{3}$. (c) The frequency-dependent factor $\mathcal{F}_{l m}\left(k_{0}\right)$, from Eq. (24), which describes the strength of the acoustic body force $f_{\text {ac }}$, when the modes of same color in (a) and (b) are combined.

This means that the optimal frequency is the center frequency between the modes, and the optimal mode separation is $\frac{1}{\sqrt{3}}$ of the full-width at half-maximum. Inserting the optimal conditions (29a) and (29b) into Eq. (28) reveals the phase difference between the modes at the optimal conditions, a value different from $\pm \frac{1}{2} \pi$,

$$
\left(\phi_{F}^{m l 0}-\phi_{F}^{l m 0}\right)^{\mathrm{opt}}= \pm \frac{1}{3} \pi .
$$

By using Eq. (26), we translate the optimal mode separation $\tilde{\Delta}_{l m}^{\text {opt }}$ into the optimal size of the symmetry breaking $d_{l m}^{\text {opt }}=$ $\left(L_{x}-L_{y}\right)_{l m}^{\mathrm{opt}}$ and the corresponding aspect ratio $A_{l m}^{\mathrm{opt}}=\left(\frac{L_{x}}{L_{y}}\right)_{l m}^{\mathrm{opt}}$, where the acoustic body force $\boldsymbol{f}_{\mathrm{ac}}$ of the double mode $\operatorname{lm} 0+$ $m l 0$ is maximized:

$$
\begin{aligned}
& d_{l m}^{\mathrm{opt}}=\tilde{\Delta}_{l m}^{\mathrm{opt}} \frac{l^{2}+m^{2}}{m^{2}-l^{2}} \Gamma_{l m}^{\star} L^{\star}, \\
& A_{l m}^{\mathrm{opt}}=1+\frac{1}{L^{\star}} d_{l m}^{\mathrm{opt}} .
\end{aligned}
$$

For all the double modes shown in Fig. 2, we have $l>m$, so for a nonrotating actuation, the rotation direction of the body force will therefore be as shown in the case of $d<0$ (corresponding to $L_{x}<L_{y}$ and $A<1$ ), and opposite for $d>0$. Note from Eq. (29e) that this asymmetry-induced rotating acoustics is extremely sensitive to $d$. In practice, it may be difficult to control this mechanism, as it requires a fabrication accuracy
TABLE II. Material parameters for water [45] and pyridine $\left(\mathrm{C}_{5} \mathrm{H}_{5} \mathrm{~N}\right)$ [46] at $25^{\circ} \mathrm{C}$ used in the numerical simulations.

\begin{tabular}{lcccc}
\hline \hline Parameter & Symbol & Water & Pyridine & Unit \\
\hline Mass density & $\rho_{\mathrm{fl}}$ & 997.05 & 982 & $\mathrm{~kg} \mathrm{~m}^{-3}$ \\
Compressibility & $\kappa_{\mathrm{fl}}$ & 448 & 507 & $\mathrm{TPa}^{-1}$ \\
Speed of sound & $c_{\mathrm{fl}}$ & 1496.7 & 1417 & $\mathrm{~m} \mathrm{~s}^{-1}$ \\
Dynamic viscosity & $\eta_{\mathrm{fl}}$ & 0.890 & 0.879 & $\mathrm{mPa} \mathrm{s}$ \\
Bulk viscosity & $\eta_{\mathrm{fl}}^{\mathrm{b}}$ & 2.485 & 62.4 & $\mathrm{mPa} \mathrm{s}$ \\
\hline \hline
\end{tabular}

much smaller than $d / L^{\star} \sim \Gamma_{l m}^{\star}$, which in microfluidic devices typically is smaller than $1 \%$ [42].

\section{3D SIMULATION OF ACOUSTIC STREAMING IN A NEARLY SQUARE CAVITY}

In this section we validate by direct numerical simulation the theory for rotating acoustics induced by a nonrotating actuation in a weakly symmetry-broken geometry. We simulate the acoustic pressure $p_{1}$ and the acoustic streaming $\boldsymbol{v}_{2}$ in a shallow, nearly square cavity in three dimensions (3D). We choose a cavity similar to that investigated experimentally by Hagsäter et al. [39], and simulated in 3D using the hard-wall approximation by Lei et al. [43], and including the actuator and elastic walls by Skov et al. [44]. The cavity has the mean side length $L^{\star}=2000 \mu \mathrm{m}, L_{x}=L^{\star}+\frac{1}{2} d, L_{y}=L^{\star}-$ $\frac{1}{2} d$, and height $L_{z}=200 \mu \mathrm{m}$. We use Eq. (29) to optimize the symmetry breaking $d$ and actuation frequency $f=\frac{1}{2 \pi} k_{0} c_{\mathrm{fl}}$ to obtain the strongest possible rotating acoustic streaming. The coordinate system is the same as in Sec. III, with $0 \leqslant x \leqslant L_{x}$, $0 \leqslant y \leqslant L_{y}$, and $0 \leqslant z \leqslant L_{z}$. To mimic an actuation from a broad piezoelectric transducer, the boundary is taken to be stationary everywhere, except at the bottom boundary $z=0$, where we apply the nonrotating Gaussian displacement in the normal direction $z$ :

$$
\begin{aligned}
\boldsymbol{u}_{1} & = \begin{cases}u_{1 z}^{0} G_{1}(x, y) \boldsymbol{e}_{z} & \text { for } z=0, \\
\mathbf{0} & \text { otherwise, }\end{cases} \\
G_{1}(x, y) & =\exp \left[-\frac{\left(x-\frac{L_{x}}{2}\right)^{2}}{\left(\frac{L_{x}}{2}\right)^{2}}-\frac{\left(y-\frac{L_{y}}{2}\right)^{2}}{\left(\frac{L_{y}}{2}\right)^{2}}\right] .
\end{aligned}
$$

This actuation displacement has the maximum amplitude of $u_{1 z}^{0}$ in the cavity center and the minimum amplitude of $e^{-2} u_{1 z}^{0} \approx 0.14 u_{1 z}^{0}$ in the corners of the cavity. In Secs. VI C $1-$ VIC 4 the fluid is water, and in Sec. VIC 6 it is pyridine. The material parameters for these fluids are listed in Table II.

In the following simulations, we excite the double-mode resonance $l 00+0 l 0$ with $l=2,4$, and 6 . Using our analytical expressions (17), we have in Fig. 2 shown the spatial dependency of the acoustic body force $\boldsymbol{f}_{\mathrm{ac}}=\boldsymbol{f}_{0 l 0}^{l 00}$ for these particular double modes. As seen in Eq. (7), this body force is the source of the bulk-driven acoustic streaming, so we predict that the numerical simulation of the bulk-driven streaming will result in a $2 \times 2$ pattern as in Fig. 2(c) for $l=2$, a $4 \times 4$ pattern as in Fig. 2(h) for $l=4$, and similarly for $l=6$ (not shown in Fig. 2). 


\section{A. Choosing the parameters}

We derived in Sec. III analytical expressions for the acoustic resonance modes including the viscous boundary layers, and we derived in Sec. V the conditions on the geometry of a nearly square cavity for which the body force $\boldsymbol{f}_{\mathrm{ac}}$ and the acoustic rotation are maximized for a nonrotating actuation. Remarkably, these analytic results allow an analytical determination of the parameter values that lead to the strongest rotating acoustic streaming in the $x y$ plane.

For the double mode $l 00+0 l 0$, the small damping coefficients $\Gamma_{\mathrm{fl}}^{l 00} \approx \Gamma_{\mathrm{fl}}^{0 l 0}$ in Eq. (4) and $\Gamma_{\mathrm{bl}}^{l 00} \approx \Gamma_{\mathrm{bl}}^{0 l 0}$ in Eq. (10b) are computed by approximating the angular frequency by the constant value $\omega=c_{\mathrm{fl}} k_{l 0}^{\star} \approx c_{\mathrm{fl}} \frac{l \pi}{L^{\star}}$ [see Eq. (25b)]:

$$
\begin{aligned}
& \Gamma_{\mathrm{fl}}^{l 00} \approx\left(\frac{4}{3}+\frac{\eta_{\mathrm{fl}}^{\mathrm{b}}}{\eta_{\mathrm{fl}}}\right) \frac{\eta_{\mathrm{fl}}}{\rho_{\mathrm{fl}} c_{\mathrm{fl}}} \frac{l \pi}{L^{\star}} \approx \Gamma_{\mathrm{fl}}^{0 l 0}, \\
& \Gamma_{\mathrm{bl}}^{l 00} \approx \frac{\delta}{L^{\star}}+\frac{\delta}{L_{z}} \approx \Gamma_{\mathrm{bl}}^{0 l 0}, \quad \delta \approx \sqrt{\frac{2 \eta_{\mathrm{fl}} L^{\star}}{l \pi c_{\mathrm{fl}} \rho_{\mathrm{fl}}}} .
\end{aligned}
$$

Combining Eqs. (10a), (29a), and (25b), we obtain an expression for the boundary-layer shifted, optimal double-mode actuation frequency, which is the center frequency of the two single modes

$$
f_{l 0}^{\star} \approx \frac{l}{2}\left(1-\frac{1}{2} \Gamma_{\mathrm{bl}}^{l 00}\right) \frac{c_{\mathrm{fl}}}{L^{\star}} .
$$

The optimal symmetry breaking $d_{l 0}^{\mathrm{opt}}$ and the corresponding aspect ratio $A_{l 0}^{\mathrm{opt}}$ then follow from Eqs. (29e) and (29f):

$$
\begin{aligned}
& d_{l 0}^{\mathrm{opt}} \approx \frac{-1}{\sqrt{3}}\left(\Gamma_{\mathrm{bl}}^{l 00}+\Gamma_{\mathrm{fl}}^{l 00}\right) L^{\star}, \\
& A_{l 0}^{\mathrm{opt}} \approx 1-\frac{1}{\sqrt{3}}\left(\Gamma_{\mathrm{bl}}^{l 00}+\Gamma_{\mathrm{fl}}^{l 00}\right) .
\end{aligned}
$$

Finally, we set the pressure amplitudes $P_{1}^{l 00}=P_{1}^{0 l 0}=1 \mathrm{MPa}$ in Eq. (12b), and compute the corresponding amplitude $u_{1 z}^{0}$ of the actuation displacement in Eq. (30) as

$$
u_{1 z}^{0}=\frac{L_{x} L_{y} L_{z}\left(\Gamma_{\mathrm{fl}}^{l 00}+\Gamma_{\mathrm{bl}}^{l 00}\right) \kappa_{\mathrm{fl}} P_{1}^{l 00}}{2 \int_{0}^{L_{x}} \int_{0}^{L_{y}} G_{1}(x, y) \cos \left(\frac{l \pi}{L^{\star}} x\right) d x d y} .
$$

For water and $l=2,4$, and 6 , the values of the expressions in Eq. (31)-(34) are listed in Table III. For pyridine, we adjust the actuation amplitude and actuation frequency to maintain the resonance amplitudes of $P_{1}^{l 00}=P_{1}^{0 l 0}=1 \mathrm{MPa}$. Note that for pyridine, we use the same optimal symmetry breaking $d_{l 0}^{\text {opt }}$ as used for water. Combining Eqs. (24), (28) and (29e) with the material parameters in Table II leads to the values $\mathcal{F}_{l 0}=0.65,0.64$, and 0.63 for $l=2,4$, and 6 , respectively, which are nearly the same as the optimal value $\mathcal{F}_{l 0}^{\mathrm{opt}}=0.65$ given in Eq. (29c).

\section{B. Implementation in COMSOL}

As in our previous work [24,44,47], the simulation is performed in two steps using the weak form PDE module in the finite-element-method software COMSOL MULTIPHYSICS [48]. In the first step, we solve for the acoustic pressure $p_{1}$ by implementing Eq. (3) in weak form, and in the second step we solve Eq. (7) for the steady streaming $\boldsymbol{v}_{2}$ and pressure $p_{2}$.

\begin{tabular}{|c|c|c|c|c|c|}
\hline Parameter & Eq. & Unit & $l=2$ & $l=4$ & $l=6$ \\
\hline \multicolumn{6}{|c|}{ Geometry (optimized for water) } \\
\hline$d_{l 0}^{\mathrm{opt}}$ & Eq. (33a) & $\mu \mathrm{m}$ & -3.922 & -2.785 & -2.286 \\
\hline$A_{l 0}^{\mathrm{opt}}$ & Eq. (33b) & 1 & 0.9980 & 0.9986 & 0.9989 \\
\hline$L^{\star}$ & & $\mu \mathrm{m}$ & 2000 & 2000 & 2000 \\
\hline$L_{z}$ & & $\mu \mathrm{m}$ & 200 & 200 & 200 \\
\hline \multicolumn{6}{|c|}{ Dependent parameters (water) } \\
\hline$u_{1 z}^{0}$ & Eq. (34) & $\mathrm{nm}$ & 1.450 & 7.474 & 14.027 \\
\hline$f_{l 0}^{\star}$ & Eq. (32) & $\mathrm{MHz}$ & 0.747 & 1.494 & 2.243 \\
\hline$\Gamma_{\mathrm{fl}}^{l 00} \approx \Gamma_{\mathrm{fl}}^{0 l 0}$ & Eq. (31a) & $10^{-3}$ & 0.008 & 0.015 & 0.023 \\
\hline$\Gamma_{\mathrm{bl}}^{l 00} \approx \Gamma_{\mathrm{bl}}^{0 l 0}$ & Eq. (31b) & $10^{-3}$ & 3.389 & 2.396 & 1.957 \\
\hline \multicolumn{6}{|c|}{ Dependent parameters (pyridine) } \\
\hline$u_{1 z}^{0}$ & Eq. (34) & $\mathrm{nm}$ & 1.757 & 9.665 & 19.617 \\
\hline$f_{l 0}^{\star}$ & Eq. (32) & $\mathrm{MHz}$ & 0.707 & 1.415 & 2.123 \\
\hline$\Gamma_{\mathrm{fl}}^{l 00} \approx \Gamma_{\mathrm{fl}}^{0 l 0}$ & Eq. (31a) & $10^{-3}$ & 0.144 & 0.287 & 0.431 \\
\hline$\Gamma_{\mathrm{bl}}^{l 00} \approx \Gamma_{\mathrm{bl}}^{0 l 0}$ & Eq. (31b) & $10^{-3}$ & 3.488 & 2.467 & 2.014 \\
\hline
\end{tabular}

TABLE III. Parameters used in the numerical simulations of the double modes $l 00+0 l 0$. The geometry parameters are optimized for maximum acoustic rotation in water, and the resulting dependent parameters (damping factors and mode amplitudes) are listed for both water and pyridine.

The zero level of $p_{2}$ is fixed by using the global constraint $\int_{\Omega} p_{2} d V=0$. Excerpts of the COMSOL code that we use are given in Ref. [44]. For numerical efficiency, we exploit the symmetry at the vertical planes $x=\frac{1}{2} L_{x}$ and $y=\frac{1}{2} L_{y}$, where we apply the symmetry conditions $\boldsymbol{n} \cdot \nabla p_{1}=0, \boldsymbol{n}$. $\boldsymbol{v}_{2}=0$, and $\boldsymbol{n} \cdot \boldsymbol{\nabla} \boldsymbol{v}_{2 \|}=\mathbf{0}$. The computational domain is thus reduced to the quadrant $0 \leqslant x \leqslant \frac{1}{2} L_{x}$ and $0 \leqslant y \leqslant \frac{1}{2} L_{y}$. We use quartic-, cubic-, and quadratic-order Lagrangian shape functions for $p_{1}, \boldsymbol{v}_{2}$, and $p_{2}$, respectively, and with a tetrahedral finite-element mesh of mesh size $\frac{1}{6} L_{z}$, we obtain $1.3 \times$ $10^{6}$ degrees of freedom and a relative accuracy better than $1 \%$. The simulations were performed on a workstation with a 3.5-GHz Intel Xeon CPU E5-1650 v2 dual-core processor, and with a memory of 128 GB RAM.

\section{Simulation results}

\section{Acoustic pressure}

In Fig. 4, we show the simulation results $p_{1}^{\text {num }}$ of the acoustic pressure $p_{1}$ in water for $l=6$ in 3D and line plots for $l=2,4$, and 6 . These three modes are examples of rotating double modes actuated by the wide, single, nonrotating Gaussian actuation $G_{1}$ [Eqs. (30) and (34)] at the bottom boundary $z=0$. In the Supplemental Material [41] we show the rotating dynamics of the pressure contours similar to Fig. 4(a) for $l=2,4$, and 6 as gif animations. In Fig. 4 we also show the analytical result $p_{1}^{\text {ana }}=p_{0 l 0}^{l 00}=p_{1}^{l 00}+p_{1}^{0 l 0}$, which is obtained from Eqs. (12) and (15) with the optimal mode separation $k_{0}^{l 00}-k_{0}^{0 l 0}=\frac{1}{\sqrt{3}} k_{l 0}^{\star} \Gamma_{l 0}^{\star}[$ Eq. (29b)] and mode amplitudes $P_{1}^{l 00}=P_{1}^{0 l 0}=1 \mathrm{MPa}$ :

$$
p_{1}^{\mathrm{ana}}=p_{0 l 0}^{l 00}=1 \mathrm{MPa} \times\left[\frac{\cos \left(\frac{l \pi x}{L^{\star}}\right)}{-\frac{1}{\sqrt{3}}+i}+\frac{\cos \left(\frac{l \pi y}{L^{\star}}\right)}{\frac{1}{\sqrt{3}}+i}\right] .
$$




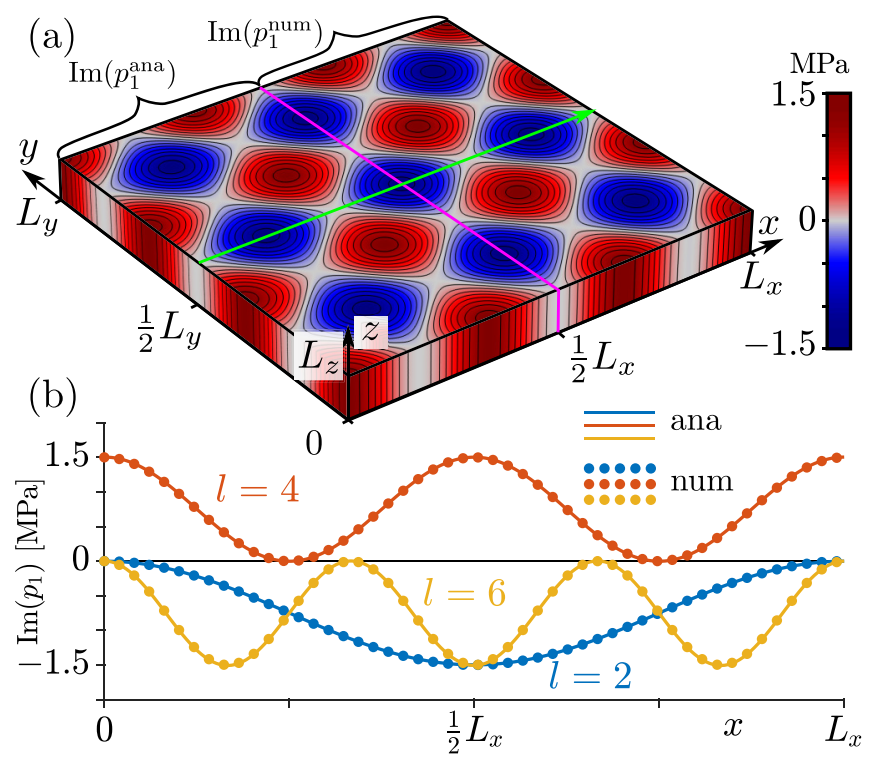

FIG. 4. The acoustic pressure $p_{1}$ for water in the nearly square cavity with parameters given in Tables II and III, where the double mode $l 00+0 l 0$ is excited by the nonrotating actuation $G_{1}$ [Eqs. (30) and (34)]. (a) Pressure contour plot for $l=6$ of the analytic solution $\operatorname{Im}\left(p_{1}^{\text {ana }}\right)$ [Eq. (35)] (for $x<\frac{1}{2} L_{x}$ ), and the numerical solution $\operatorname{Im}\left(p_{1}^{\text {num }}\right)$ (for $\left.x>\frac{1}{2} L_{x}\right)$. (b) Line plots of the pressure $\operatorname{Im}\left(p_{1}^{\text {ana }}\right)$ (solid) and $\operatorname{Im}\left(p_{1}^{\text {num }}\right)$ (dots) along the green line in (a) at $y=\frac{1}{2} L_{y}$ and $z=L_{z}$ for the modes $l=2$ (blue), $l=4$ (orange), and $l=6$ (yellow). Animations of the rotating acoustic fields are shown in the Supplemental Material [41].

In Fig. 4(b), we compare $\operatorname{Im}\left(p_{1}^{\text {num }}\right)$ and $\operatorname{Im}\left(p_{1}^{\text {ana }}\right)$ and find a quantitative agreement better than $1 \%$. The reason for choosing the imaginary part is to single out the resonant part of the field, which is phase shifted by the factor $\exp \left(i \frac{\pi}{2}\right)=i$ relative to the actuation displacement $\boldsymbol{u}_{1}$ at the boundary Eq. (30). All nonresonant contributions are in phase with $\boldsymbol{u}_{1}$. In the Supplemental Material [41] is shown an animated gif file $^{2}$ of the full cycle in the time domain of the line plots in Fig. 4(b) of $p_{1}$. These animations reveal that the deviation of the analytical solution from the numerical one is $\sim 1 \%$ for $l=2$ at $0.75 \mathrm{MHz}, \sim 15 \%$ for $l=4$ at $1.5 \mathrm{MHz}$, and $\sim 30 \%$ for $l=6$ at $2.2 \mathrm{MHz}$. The deviation increases for increasing mode number because the wide vertical $G_{1}$ actuation couples strongly to the vertical 001 mode through the amplitude factor $P_{1}^{001}$ of Eq. (12b), while the frequency-dependency factor $F^{001}\left(k_{0}\right)$ of Eq. $(12 \mathrm{c})$, which is very small away from resonance, increases significantly as the actuation frequency $f=c_{\mathrm{fl}} k_{0}$ approaches the vertical-mode resonance $f_{0}^{001}=3.7$ $\mathrm{MHz}$. We can reduce the coupling $P_{1}^{001}$ to the vertical 001 mode by reducing the width of the $G_{1}$ actuation profile. This is demonstrated in the animated gif file ${ }^{3}$ of the Supplemental Material [41], where the width of the $G_{1}$ actuation has been reduced by a factor of 6 from $\frac{1}{2} L^{\star}$ to $\frac{1}{12} L^{\star}$. This results in a decreased deviation, now less than $\sim 1 \%$, of the analytical result from the numerical one for $p_{1}$ for all three modes $l=2,4,6$.

\footnotetext{
2"p1_246_wide_G1.gif"

3"p1_246_narrow_G1.gif"
}

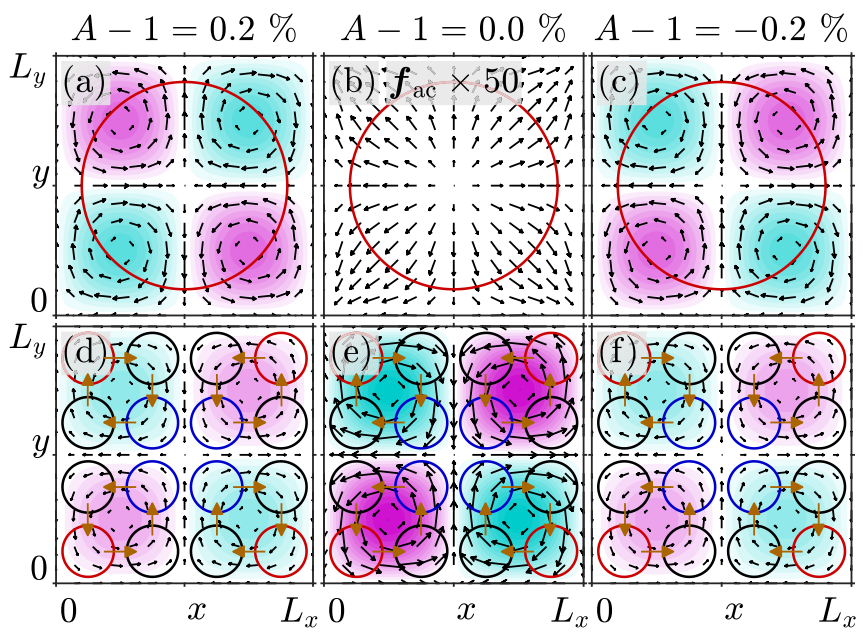

FIG. 5. Simulation of the rotating acoustics in the $x-y$ plane for the double mode $200+020$ in water for three aspect ratios $A \approx 1.002,1,0.998$ each for the nonrotating $\left(G_{1}\right)$ and rotating $\left(G_{16}\right)$ actuation perpendicular to the plane. Color plots between $\pm 10^{-4} \mathrm{~kg} \mathrm{~m}^{-1} \mathrm{~s}^{-1}$ (light cyan to dark magenta) of the $z$ component $\mathcal{L}_{\mathrm{ac}, z}$ of the angular momentum density $\mathcal{L}_{\mathrm{ac}}$, and vector plots of the acoustic body force $f_{\text {ac }}\left(\max 2.00 \mathrm{Nm}^{-3}\right)$. In (a)-(c) we use the single nonrotating Gaussian actuation $G_{1}$ (large red circles) [Eqs. (30) and (34)]. In (d)-(f) we use the rotating actuation profile $G_{16}$ of four quadruples of phase shifted Gaussian profiles given in Eq. (C1) of Appendix C [light red (up), black (0), dark blue (down), and black (0) circles, respectively] to generate an actuation rotating in the direction of the thick brown arrows. $A \approx 1.002$ and 0.998 are chosen to optimize the acoustic rotation for the nonrotating actuation [see Eq. (29)].

\section{Acoustic body force and angular momentum density}

In Fig. 5, we validate our analysis in Sec. V of rotating double modes induced by a nonrotating actuation. For the double mode $200+020$, we plot the body force $\boldsymbol{f}_{\mathrm{ac}}=\boldsymbol{f}_{020}^{200}$ from Eq. (17), which according to Eq. (7) is the source of the bulk-driven acoustic streaming, and the acoustic angular momentum density $\mathcal{L}_{\mathrm{ac}}=\mathcal{L}_{020}^{200}$ from Eq. (18b) in the center plane $z=\frac{1}{2} L_{z}$. We show results for three different cavity aspect ratios $A=L_{x} / L_{y}$ for both the nonrotating wide Gaussian actuation $G_{1}(x, y)$, given in Eq. (30), and for the externally controlled rotating actuation $G_{16}(x, y)$ consisting of four quadruples of narrow Gaussian actuations [see Eq. (C1) of Appendix C]. As expected, the nonrotating actuation $G_{1}$ used in Figs. 5(a)-5(c) induces no acoustic rotation for a perfect square cavity $A=1$ [Fig. 5(b)], while it induces different rotation directions for $A$ slightly larger and smaller than unity [see Figs. 5(a) and 5(c), respectively]. In contrast, the rotating actuation $G_{16}$ used in Figs. 5(d)-5(f) for the same three aspect ratios, induces an acoustic rotation, which is maximized in the perfect square cavity $A=1$ [see Fig. 5(e)]. We show in Eq. (C4) of Appendix $C$ that this rotating actuation excites the modes 200 and 020 with a phase difference $\phi_{\text {act }}^{020}-\phi_{\text {act }}^{200} \approx$ $0.5 \pi$ such that the factor $\mathcal{F}_{020}^{200}$ in Eq. (19) is positive, and consequently the rotation pattern in Figs. 5(d)-5(f) is in the same direction as shown in Fig. 2(c).

In Fig. 6 we investigate the strength and rotation direction of the body force $f_{020}^{200}$ as a function of the mode separation 


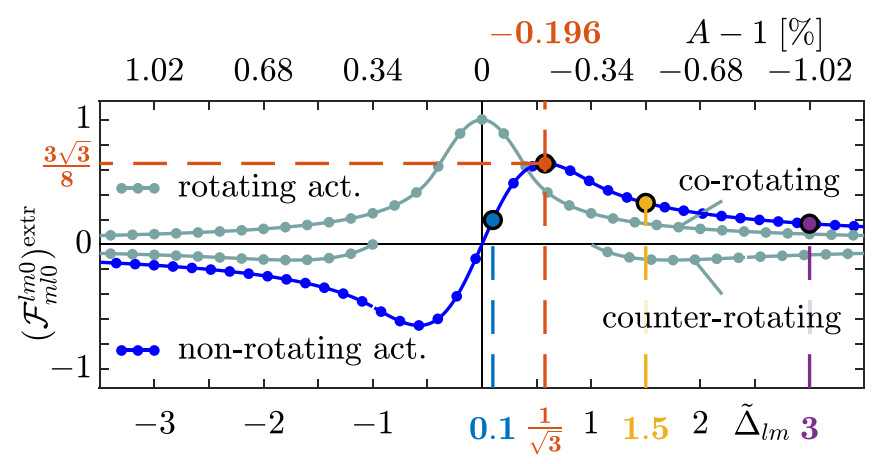

FIG. 6. The largest possible value (positive and negative) of $\mathcal{F}_{m l 0}^{\operatorname{lm} 0} \propto \boldsymbol{f}_{\mathrm{ac}}$ versus varying mode separation $\tilde{\Delta}_{l m}$ (lower $x$ axis) or aspect ratio $A$ (upper $x$ axis), shown for nonrotating actuations (blue) and rotating actuations (light cyan). The numerical values (dots) are found by evaluating $\left(\mathcal{F}_{020}^{200}\right)_{\text {num }}$ from Eq. (36) in the simulation of the double mode $200+020$ shown in Figs. 5(a)-5(c) for the nonrotating and Figs. 5(d)-5(f) for the rotating actuation. The analytical curves (solid) are from the expressions derived in Eq. (B4a) (nonrotating act.), Eq. (B7a) (rotating act., corotating), and Eq. (B8a) (rotating act., counter-rotating) of Appendix B. The black-edged circles correspond to those of the same color in Fig. 3(c).

$\tilde{\Delta}_{20}$ or, equivalently, the aspect ratio $A$. From Eq. (17a), this dependency is quantified through the factor $\mathcal{F}_{020}^{200}$ which we obtain numerically from the simulation of the double mode $200+020$ in Fig. 5 by calculating the acoustic angular momentum $\mathcal{L}_{\text {ac }}$ and using Eq. (18b):

$$
\left(\mathcal{F}_{020}^{200}\right)_{\mathrm{num}} \approx \frac{\mathcal{L}_{\mathrm{ac}, z}\left(\frac{1}{4} L_{x}, \frac{1}{4} L_{y}\right)}{\left|P_{1}^{200}\right|\left|P_{1}^{020}\right|\left(\omega \rho_{\mathrm{ff}} c_{0}^{2}\right)^{-1}} .
$$

Here, for pressure amplitudes $\left|P_{1}^{200}\right|=\left|P_{1}^{020}\right|=1 \mathrm{MPa}$, the denominator evaluates to $0.954 \times 10^{-4} \mathrm{~kg} \mathrm{~m}^{-1} \mathrm{~s}^{-1}$, which is indeed the maximum value found numerically in Fig. 5(e), such that $\left(\mathcal{F}_{020}^{200}\right)_{\text {num }}$ takes the maximum value of unity, as expected. In Fig. 6 we plot the largest possible value of $\left(\mathcal{F}_{020}^{200}\right)_{\text {num }}$ both for the nonrotating actuation used in Figs. 5(a)-5(c) and for the rotating actuation used in Figs. 5(d)-5(f). We also plot the analytical expressions for $\left(\mathcal{F}_{m l 0}^{l m 0}\right)_{\text {ana }}^{\text {extr }}$ derived in Appendix B and find a quantitative agreement better than $1 \%$. For the nonrotating actuation (the dark blue curve in Fig. 6), the acoustic rotation direction is determined by the aspect ratio $A$, and it reverses as $A$ crosses over from $A<1$ to $A>1$ around the perfect square $A=1$. For the rotating actuation (the light blue curves in Fig. 6), the rotation direction can be either corotating or counter-rotating (at different frequencies) with respect to the rotation direction of the actuation. However, for small mode separations $\left|\tilde{\Delta}_{l m}\right|<1$, which for the double mode $200+020$ corresponds to $|A-1|<0.34 \%$, the rotation direction of the acoustic field can only be corotating. For further details, see Appendix B.

\section{Acoustic streaming}

In Fig. 7, we show the acoustic streaming velocity $\boldsymbol{v}_{2}$ in 3D resulting from the double mode $600+060$ displayed in Fig. 4(a), which is excited by the nonrotating actuation $G_{1}$. As mentioned above, this situation is similar to that investigated

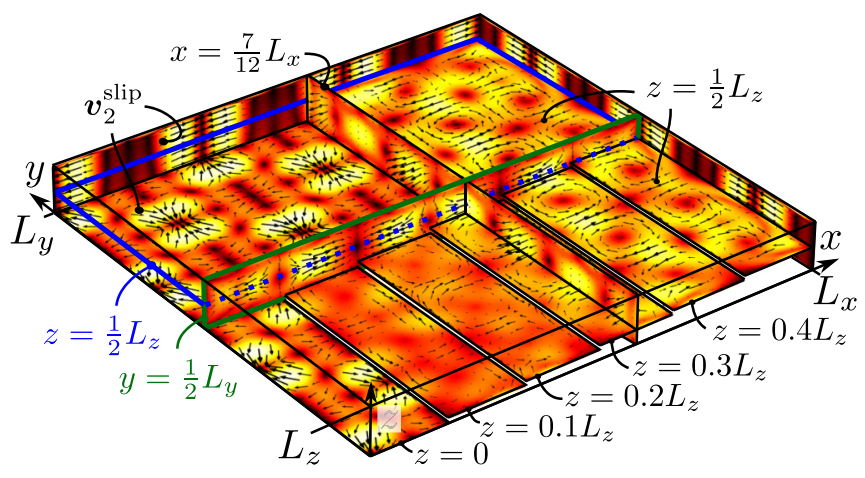

FIG. 7. Numerical simulation of the acoustic streaming velocity $\boldsymbol{v}_{2}$ (black arrows) and its magnitude from 0 (black) to $0.17 \mathrm{~mm} / \mathrm{s}$ (white) induced by the nonrotating actuation $G_{1}$. The setup and the parameters are the same as in Fig. 4 with the parameters listed in Tables II and III. The horizontal plane at $z=\frac{1}{2} L_{z}$ (dark blue edge) and the vertical plane at $y=\frac{1}{2} L_{y}$ (light green edge) are displayed in Fig. 8.

experimentally by Hägsater et al. [39]. Remarkably, in the optimized geometry of Fig. 7, the horizontal $6 \times 6$ streamingroll pattern at any height $0 \leqslant z \leqslant L_{z}$ is strongest in the center of the cavity, $z=\frac{1}{2} L_{z}$, thus indicating its bulk-driven origin. In the bottom plane $z=0$ (shown in Fig. 7) and in the top plane $z=L_{z}$ (not shown in Fig. 7), the slip velocity $\boldsymbol{v}_{2}^{\text {slip }}$ is dominated by the gradient term in the expression (7e) for the slip velocity, and has only a weak rotating component.

In Fig. 8, we compare the results for the acoustic streaming $\boldsymbol{v}_{2}$ [see Eq. (7)] for the double modes $l 00+0 l 0$ with $l=$ $2,4,6$. The streaming is shown in the horizontal $x-y$ center plane at height $z=\frac{1}{2} L_{z}$ (dark blue edge in Fig. 7) and in the vertical $x-z$ center plane at $y=\frac{1}{2} L_{y}$ (light green edge in Fig. 7). In the first row [Figs. 8(a)-8(c)] we show the bulk-driven streaming alone ( $\boldsymbol{f}_{\mathrm{ac}}$ on, $\boldsymbol{v}_{2}^{\text {slip }}$ off); in the second row [Figs. 8(d)-8(f)] we show the boundary-driven streaming alone $\left(\boldsymbol{f}_{\text {ac }}\right.$ off, $\boldsymbol{v}_{2}^{\text {slip }}$ on); in the third row [Figs. 8(g)-8(i)] we show the total streaming ( $\boldsymbol{f}_{\mathrm{ac}}$ on, $\boldsymbol{v}_{2}^{\text {slip }}$ on). Comparing the bottom-row and top-row panels in Fig. 8 strongly indicate that the respective $4 \times 4$ and $6 \times 6$ streaming-roll patterns in Figs. 8(h) and 8(i) are bulk driven. Furthermore, Figs. 8(a)8(c) show that the bulk-driven acoustic streaming becomes stronger for higher mode number $l$ and thus for higher frequency $f$. In contrast, the boundary-driven acoustic streaming [Figs. 8(d)-8(f)] stays almost constant in amplitude and shows no frequency dependency. Note that the bulk-driven streaming in Figs. 8(a)-8(c) has its maximum velocity $v_{2}^{\max }$ in the bulk of the fluid whereas the boundary-driven streaming in Figs. 8(d)$8(\mathrm{f})$ has its maximum velocity $v_{2}^{\max }$ at the boundaries.

\section{Comparison with the analytical expression for the bulk-driven streaming}

When the no-slip condition on the vertical side walls is ignored, we can derive an approximate analytical expression for the bulk-driven streaming $\left(\boldsymbol{v}_{2}^{\mathrm{blk}}\right)_{l 0}$ for the double modes $l 00+0 l 0$. The details are given in Appendix D, where we find $\left(\boldsymbol{v}_{2}^{\mathrm{blk}}\right)_{l 0}$ to be proportional to the acoustic energy flux density 

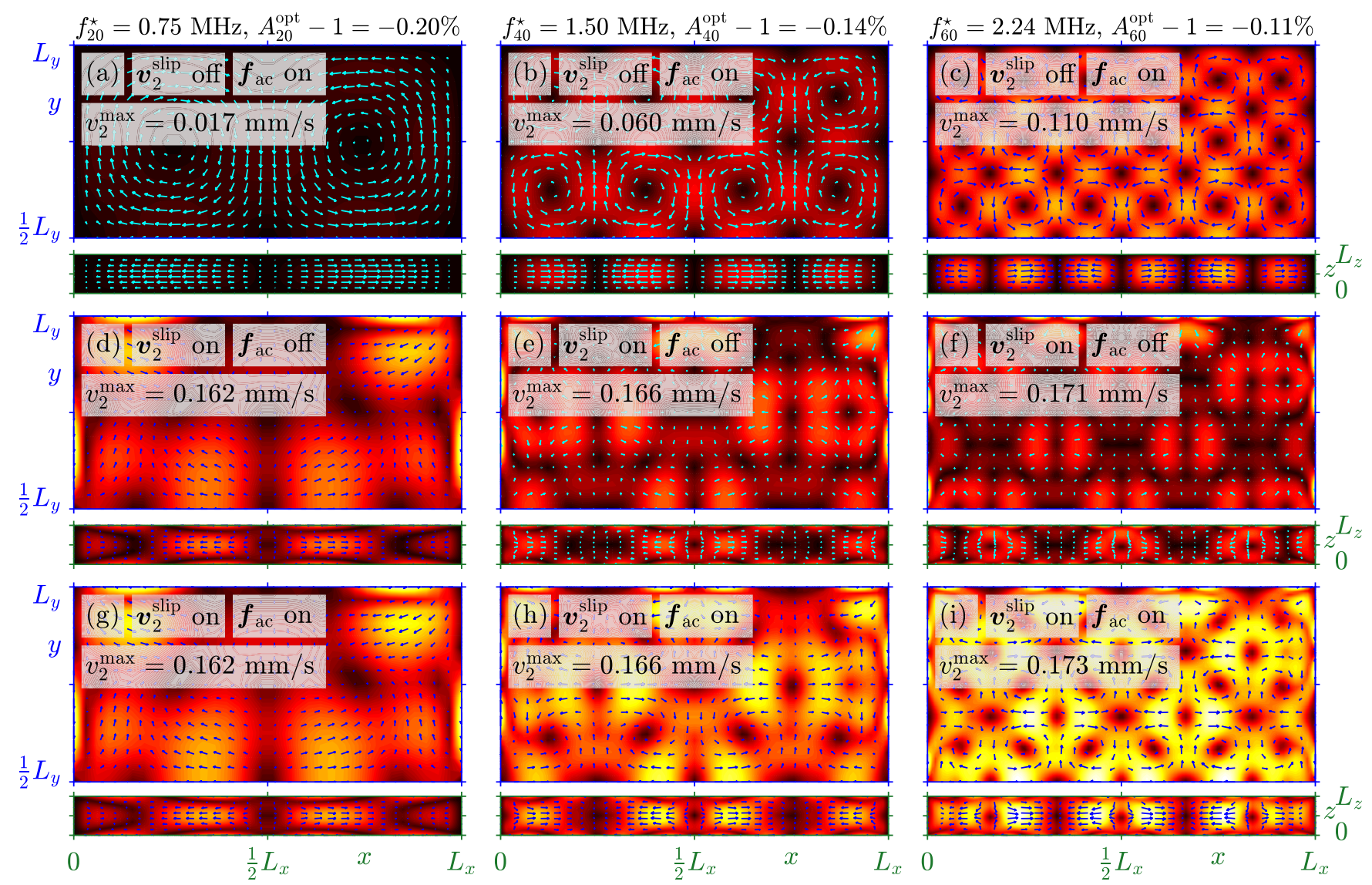

FIG. 8. Simulation of the acoustic streaming velocity $\boldsymbol{v}_{2}$ in water from 0 (black) to $0.17 \mathrm{~mm} / \mathrm{s}$ (white) in the horizontal ( $x-y$ ) center plane at $z=\frac{1}{2} L_{z}$ (dark blue edge as in Fig. 7) and the vertical $(x-z)$ center plane at $y=\frac{1}{2} L_{y}$ (light green edge as in Fig. 7). The length of the longest arrow represents $v_{2}^{\max }$ listed in each plot. The used parameters are listed in Tables II and III. In each column the double mode $l 00+0 l 0$ is excited for $l=2,4$, and 6, respectively. The first row (a)-(c) shows the bulk-driven streaming, the second row (d)-(f) shows the boundary-driven streaming, and the third row (g)-(i) shows the total streaming. 3D results corresponding to (i) are shown in Fig. 7.

$S_{l 0}=S_{0 l 0}^{l 00}$ of Eq. (18a):

$$
\left(\boldsymbol{v}_{2}^{\mathrm{blk}}\right)_{l 0}=\frac{1}{2}\left(\frac{4}{3}+\frac{\eta_{\mathrm{fl}}^{\mathrm{b}}}{\eta_{\mathrm{fl}}}\right)\left[1-\frac{\cosh \left(\frac{2 z-L_{z}}{\sqrt{2}} k_{l 0}^{\star}\right)}{\cosh \left(\frac{1}{\sqrt{2}} k_{l 0}^{\star} L_{z}\right)}\right] \kappa_{\mathrm{fl}} \boldsymbol{S}_{l 0} .
$$

Inserting here $k_{l 0}^{\star}=\frac{l \pi}{L^{\star}}$ and expression (18a) for $\boldsymbol{S}_{l 0}$ with $\mathcal{F}_{l 0}=\frac{3 \sqrt{3}}{8}$ from Eq. (29c), we obtain the maximum value of the bulk-driven streaming velocity in the center plane $z=\frac{1}{2} L_{z}$ :

$$
\left(v_{2}^{\mathrm{blk}}\right)_{l 0}^{\max }=\frac{3 \sqrt{3}\left[1-\operatorname{sech}\left(\frac{l \pi L_{z}}{\sqrt{2} L^{*}}\right)\right]\left(\frac{4}{3}+\frac{\eta_{\mathrm{f}}^{\mathrm{b}}}{\eta_{\mathrm{f}}}\right)\left|P_{1}^{l 00}\right|\left|P_{1}^{0 l 0}\right|}{32 \rho_{\mathrm{fl}}^{2} c_{\mathrm{fl}}^{3}} .
$$

For the three cases shown in Figs. 8(a)-8(c), where we have used $P_{1}^{l 00}=P_{1}^{0 l 0}=1 \mathrm{MPa}$ and $l=2,4,6$, respectively, we calculate from Eq. (38) the maximum values 0.018, 0.060, and $0.102 \mathrm{~mm} / \mathrm{s}$, which deviate less than $7 \%$ from the corresponding results $0.017,0.060$, and $0.110 \mathrm{~mm} / \mathrm{s}$ from the numerical simulation, listed in Figs. 8(a)-8(c). For $l=2$, the analytical prediction of $0.018 \mathrm{~mm} / \mathrm{s}$ overestimates by $5 \%$ the numerical value $0.017 \mathrm{~mm} / \mathrm{s}$ because it neglects the significant viscous damping from the vertical side walls in this case. For $l=6$, the analytical prediction of $0.102 \mathrm{~mm} / \mathrm{s}$ underestimates by $7 \%$ the numerical value $0.110 \mathrm{~mm} / \mathrm{s}$ because the latter includes the coupling to the vertical 001 mode through the wide $\frac{1}{2} L^{\star}$-width vertical $G_{1}$ actuation as discussed in Sec. VIC 1 . When changing to the narrow $\frac{1}{12} L^{\star}$-width vertical $G_{1}$ actuation, also discussed in Sec. VIC 1, the coupling to the vertical 001 mode diminishes so much that the numerical streaming amplitudes become $0.017,0.060$, and $0.103 \mathrm{~mm} / \mathrm{s}$ for $l=2,4,6$, respectively, where the latter now deviates less than $1 \%$ from the analytical prediction $0.102 \mathrm{~mm} / \mathrm{s}$. These numerical results for the acoustic streaming are shown in a pdf file ${ }^{4}$ in the Supplemental Material [41].

\section{Bulk-driven versus boundary-driven streaming rolls}

We address the question on whether the horizontal streaming rolls in the $x y$ plane are due to the bulk-driven streaming or the boundary-driven streaming. We restrict the discussion to horizontal double modes $l 00+0 l 0$, for which we have found the analytical expressions for the bulk-driven streaming $\left(\boldsymbol{v}_{2}^{\text {blk }}\right)_{l 0}$ [Eq. (37)] and the slip velocity $\boldsymbol{v}_{2}^{\text {slip }}$ [Eq. (7e)] at the top $\left(z=L_{z}\right)$ and bottom $(z=0)$ boundaries.

\footnotetext{
4“fig_08_narrow_G1.pdf"
} 

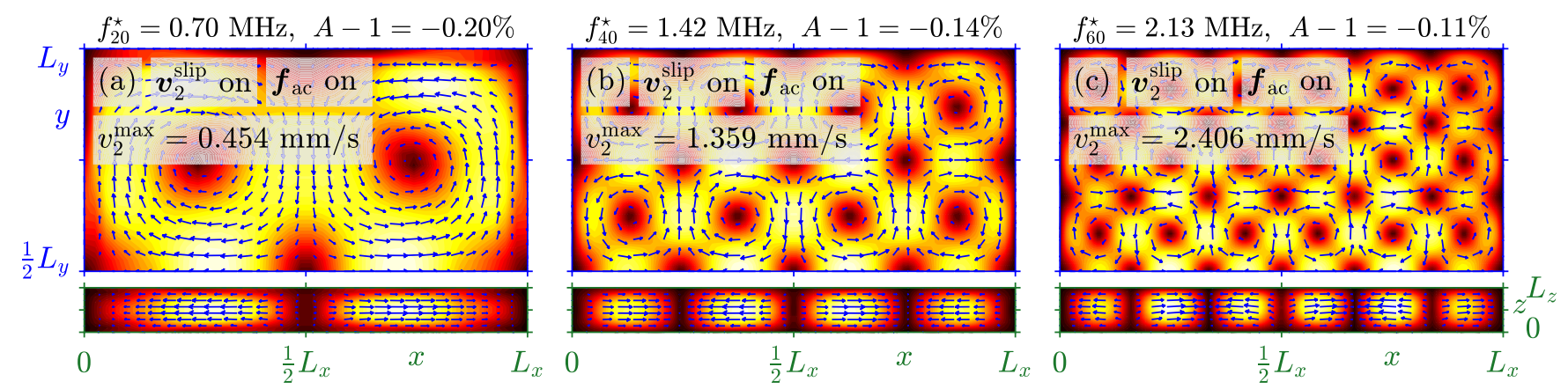

FIG. 9. Simulation of the acoustic streaming $\boldsymbol{v}_{2}$ from $0 \mathrm{~mm} / \mathrm{s}$ (black) to $v_{2}^{\max }$ (white) for the exact same geometry as in Figs. 8(g)-8(i), but with the water replaced by pyridine, having the material parameters listed in Table II and actuation amplitude $u_{1 z}^{0}$ and actuation frequency $f_{l 0}^{\star}$ given in Table III. The maximum values in (a)-(c) are 2.8, 8.2, and 13.9 times the corresponding values in Figs. 8(g)-8(i).

According to Eq. (7e), the boundary-driven streaming velocity at the double-mode resonance $l 00+0 l 0$ is driven by a slip velocity, which contains the term $\frac{1}{2} \kappa_{\mathrm{fl}} \boldsymbol{S}_{\mathrm{ac}}$ that rotates in the direction of the acoustic energy flux density $\boldsymbol{S}_{\mathrm{ac}}$, just as the bulk-driven streaming [see Eq. (37)]. The other terms in the slip velocity have similar magnitude, but they are gradient terms which cannot drive a rotating streaming in the horizontal $x y$ plane. Therefore, as seen in Figs. 8(d)-8(f), the boundary-driven streaming does not lead to horizontal flow rolls as clearly as the bulk-driven streaming does [see Figs. 8(a)-8(c)]. There may, however, exist horizontal planes at a few specific heights $z$, where the horizontal flow-roll pattern appears more clearly as suggested by Lei et al. [43] and analyzed further by Skov et al. [44].

By using Eqs. (7e) and (37), we compute the ratio of the horizontally rotating bulk-driven streaming $\left|\left[\boldsymbol{v}_{2}^{\mathrm{blk}}\left(z=\frac{L_{z}}{2}\right)\right]_{l 0}\right|$ in the center of the cavity to the horizontally rotating boundary-driven streaming $\left|\frac{1}{2} \kappa_{\mathrm{ff}} \boldsymbol{S}_{\mathrm{ac}}(z=0)\right|$ at the bottom boundary,

$$
\frac{\left|\left[\boldsymbol{v}_{2}^{\mathrm{blk}}\left(z=\frac{L_{z}}{2}\right)\right]_{l 0}\right|}{\left|\frac{1}{2} \kappa_{\mathrm{ffl}} \boldsymbol{S}_{l 0}(z=0)\right|}=\left(\frac{4}{3}+\frac{\eta_{\mathrm{fl}}^{\mathrm{b}}}{\eta_{\mathrm{fl}}}\right)\left[1-\operatorname{sech}\left(\frac{l \pi L_{z}}{\sqrt{2} L^{\star}}\right)\right] .
$$

This velocity ratio takes values from 0 (boundary-driven streaming dominates) for a flat cavity $l L_{z} \ll L^{\star}$ to $\frac{4}{3}+\frac{\eta_{n}^{b}}{n_{n}}$ (bulk-driven streaming dominates) for a high cavity $l L_{z} \gg L^{\star}$. For water, the velocity ratio (39) lies between 0 and 4.13 , and in the setup of Figs. 4-8 with $L_{z}=0.1 L^{\star}$ and water as the fluid, we obtain the values of the velocity ratio to be $0.38,1.22$, and 2.09 for $l=2,4$, and 6 , respectively. This result indicates that the $6 \times 6$ streaming roll pattern with $l=6$ observed by Hagsäter et al. [39] is predominantly bulk-driven, as also seen by comparing Figs. 8(c) and 8(i). In contrast, for the lower mode $l=2$, the horizontal streaming rolls are predominantly boundary driven, which is seen by comparing Figs. 8(d) and $8(\mathrm{~g})$.

The crossover from boundary- to bulk-driven horizontal streaming rolls may be characterized by the height-to-width ratio $B=l L_{z} / L^{\star}$ : we compute the critical value $B^{\text {crit }}$, where the velocity ratio (39) is unity, and thus,

$$
\begin{aligned}
& l \frac{L_{z}}{L^{\star}}<B^{\text {crit }}, \quad \text { boundary-driven streaming dominates, } \\
& l \frac{L_{z}}{L^{\star}}>B^{\text {crit }}, \quad \text { bulk-driven streaming dominates. }
\end{aligned}
$$

For water, Eq. (39) leads to the ratio $B_{\text {water }}^{\text {crit }}=0.35$, and since $L_{z}=0.1 L^{\star}$ in the setup of Figs. 4-8, we find that the horizontal streaming rolls in the double modes $l 00+0 l 0$ with $l \leqslant 3$ are predominantly boundary driven, while for $l \geqslant 4$ they are bulk driven.

\section{Enhancement of the bulk-driven streaming velocity by increasing the bulk viscosity}

For a given resonance mode with a given acoustic pressure amplitude in a given geometry, we obtain from Eqs. (38) and (7e) the following scaling laws for the bulk- and boundarydriven streaming, respectively:

$$
\begin{aligned}
& v_{2}^{\mathrm{blk}} \propto \frac{1}{\rho_{\mathrm{fl}}^{2} c_{\mathrm{fl}}^{3}}\left(\frac{4}{3}+\frac{\eta_{\mathrm{fl}}^{\mathrm{b}}}{\eta_{\mathrm{fl}}}\right), \\
& v_{2}^{\text {slip }} \propto \frac{1}{\rho_{\mathrm{fl}}^{2} c_{\mathrm{fl}}^{3}} .
\end{aligned}
$$

To study the consequences of these scaling laws, we have chosen to compare water with the heterocyclic organic compound pyridine having the chemical formula $\mathrm{C}_{5} \mathrm{H}_{5} \mathrm{~N}$. Table II reveals that pyridine has the same material parameters (within 5\%) as water, but a bulk viscosity $\eta_{\mathrm{fl}}^{\mathrm{b}}$ that is 25.1 times larger than that of water. Using these parameter values in Eq. (41), we find a large enhancement of the bulk-driven streaming, while the boundary-driven streaming is nearly unchanged:

$$
\begin{aligned}
& v_{2, \text { pyridine }}^{\text {blk }}=21.3 v_{2, \text { water }}^{\mathrm{blk}}, \\
& v_{2, \text { pyridine }}^{\text {slip }}=1.2 v_{2, \text { water }}^{\text {slip }} .
\end{aligned}
$$

We test this prediction in Fig. 9, where we show simulations of the total acoustic streaming in pyridine in the same geometry as in Figs. 8(g)-8(i) but with adjusted actuation amplitude $u_{1 z}^{0}$ and frequency $f$ given in Table III to maintain the same pressure amplitudes $P_{1}^{l 00}=P_{1}^{0 l 0}=1 \mathrm{MPa}$. As expected, we find that the total acoustic streaming for the three double modes is enhanced compared to that in water shown in Figs. 8(g)-8(i), and the enhancement factors are 2.8, 8.2, and 13.9 , respectively. The increasing factors reflect the increasing weight of bulk-driven relative to boundary-driven streaming as the mode index $l$ increases. The enhancement factors for the bulk-driven streaming alone (not shown in Fig. 9) are 21.4, 21.1, and 21.2, as predicted by Eq. (42a), while they are 1.2, 
1.2, and 1.3 for boundary-driven streaming alone (also not shown in Fig. 9) in good agreement with Eq. (42b).

For all three cases $l=2,4$, and 6 , the horizontally rotating bulk-driven streaming in the center $z=\frac{1}{2} L_{z}$ is larger than the horizontally rotating boundary-driven streaming at the topbottom boundaries $z=0$ and $z=L_{z}$. This is also predicted by Eq. (40) since $l \frac{L_{z}}{L^{\star}}=0.2,0.4,0.6>B_{\text {pyridine }}^{\text {crit }}=0.08$ for all three modes in pyridine.

\section{DISCUSSION}

In the following, we raise a few discussion points related to the results of the above analysis, which are based on the single- and double-mode resonances occurring in a closed rectangular-box-shaped cavity with narrow viscous boundary layers. We note that the idealized, hard-wall, numerical model for the acoustic streaming is strikingly sensitive to small differences of only $0.1 \%$ between the cavity side lengths $L_{x}$ and $L_{y}$. Our results for the resonance frequency in Eq. (10) and the optimal aspect ratio in Eq. (29f) are therefore crucial in the investigation of the largest possible bulk-driven acoustic streaming. This optimization was not taken into account in the experimental study by Hagsäter et al. [39], and the corresponding numerical simulation by Skov et al. [44] of the shallow, nearly square cavity of aspect ratio of $A=0.990$. As this aspect ratio differs from the optimal value $A=0.999$ for the hard-wall device in Fig. 8(i), it may be possible to increase the bulk-driven acoustic streaming significantly by fine tuning the geometry.

A priori, one may argue that it is difficult to obtain these optimal conditions with a relative precision of the order of $0.1 \%$ in experiments. However, comparing our Fig. 7 with the experimental results in Hagsäter et al. [39], we find good qualitative agreement even though none of the optimal conditions were considered when designing the nearly square fluid cavity. Another example is provided by the straight microchannel with a square cross section and nonrotating actuation that was studied experimentally by Antfolk et al. [11]. There, a single large vertical streaming roll similar to Fig. 2(b) was observed experimentally and analyzed numerically in terms of a double-mode excitation.

There may be several reasons for why the bulk-driven acoustic streaming can be observed experimentally in spite of the low $0.1 \%$-tolerance level of the aspect ratio suggested by our simplified, hard-wall, microcavity analysis in Fig. 6. First, there are other damping mechanisms in a real experiment [42], aside from the bulk and boundary-layer damping considered here, which may broaden the resonance peaks significantly and thereby increase the change for overlapping resonances. Second, the double-mode body force $f_{l m n}^{l m n}$ in Eq. (17a), which is the source of the bulk-driven acoustic streaming, depends on the product $\left|P_{1}^{l m n}\right|\left|P_{1}^{l^{\prime n} m}\right|$ of the mode amplitudes, and therefore one can obtain a rotating body force even if the pressure is dominated by one of the modes, say lmn, as long as the other mode $l^{\prime} m{ }^{\prime} n^{\prime}$ is just weakly represented. Third, in typical experiments, the driving frequency is scanned over a broad span of frequencies, say $100 \mathrm{kHz}$ around $2 \mathrm{MHz}$, which increases the possibility for exciting double-mode resonances. Fourth, unintentional asymmetries and imperfections in the experimental setup may cause modes to overlap locally in some regions at some frequencies and in other regions at other frequencies, rather than the idealized homogeneous pattern at a single frequency shown in Fig. 7. This point was also mentioned by Hagsäter et al:: "If the frequency is shifted slightly in the vicinity of $2.17 \mathrm{MHz}$, the same vortex pattern will still be visible, but the strength distribution between the vortices will be altered." Moreover, in the 3D simulation by Skov et al. [44], including the surrounding solid and the piezoelectric transducer, this inhomogeneous distribution of the streaming was indeed reproduced in their Fig. 2(b2). Revisiting the experiment by Antfolk et al. [11] of a long straight capillary of square cross section, this effect may also be observed because it is likely that the aspect ratio is slightly less than unity in some regions of the capillary and slightly above unity in other regions, thereby causing the rotation direction to alternate along the channel.

We have in this work mainly considered horizontal acoustics with $n=0$ half-waves in the vertical $z$ direction. A similar analysis can be done for perpendicular acoustics (with respect to the top and bottom boundaries). In Secs. VIC 1 and VIC4, we have already briefly discussed the role of the vertical single mode 001 for the pressure $p_{1}$ and the streaming $\boldsymbol{v}_{2}$. It is of course also possible to consider vertical rotating double modes involving, say, a single half-wave in the vertical direction $l m 1+l^{\prime} m$ ' 1 . For relatively shallow cavities, a vertical excitation implies a relatively high frequency and a correspondingly increased body force $\boldsymbol{f}_{\mathrm{ac}}$. Furthermore, for perpendicular acoustics where $\partial_{\perp} v_{1 \perp} \approx \nabla \cdot v_{1}=i \omega \kappa_{\mathrm{fl}} p_{1}$, the slip velocity $\boldsymbol{v}_{2}^{\text {slip }}$ on the top and bottom boundaries is dominated by the first and last terms in Eq. (7e), $\boldsymbol{v}_{2}^{\text {slip }} \approx \frac{1}{2} \kappa_{\mathrm{ff}} \boldsymbol{S}_{\mathrm{ac}}+$ $\frac{3}{4 \omega} \operatorname{Re}\left[i \partial_{\perp} v_{1 \perp} v_{1 \|}^{*}\right] \approx-\kappa_{\mathrm{fl}} \boldsymbol{S}_{\mathrm{ac}}$. Remarkably, this slip velocity is in the opposite direction of the body force $f_{\mathrm{ac}} \propto S_{\mathrm{ac}}$, so we expect that the bulk- and boundary-driven streaming will be in opposite directions, and thus the cavity could be designed such that these streaming flows tend to cancel. A study of this effect is left for future work.

The bulk-driven acoustic streaming is due to the body force $\boldsymbol{f}_{\mathrm{ac}}$, which we have given in Eq. (7d) for homogeneous fluids. As shown by Karlsen and Bruus [49], this body force contains additional terms if the fluid is inhomogeneous. Furthermore, the analysis by Muller and Bruus [45] showed how thermoviscous effects strongly influence the boundary-driven streaming. The incorporation of inhomogeneities and thermodynamics in future work is therefore expected to reveal a strong effect also on the bulk-driven streaming.

\section{CONCLUSION}

We have derived analytical expressions for the singlemode pressure resonances $p_{1}^{l m n}=P_{1}^{l m n} F^{l m n} R^{l m n}$ and the corresponding boundary-layer damping coefficients $\Gamma_{\mathrm{bl}}^{l m n}$ for a closed rectangular-box-shaped cavity with narrow viscous boundary layers (see Sec. III A and Appendix A). Based on these expressions, we have shown that the body force $\boldsymbol{f}_{\mathrm{ac}}$ can drive a strong bulk-driven acoustic streaming, if two overlapping single modes are excited simultaneously at the same frequency, thereby forming a double-mode resonance $l m n+l^{\prime} m n^{\prime}$ (see Secs. III B and IV). In contrast to the conventional wisdom that the bulk-driven streaming can be ignored 
on the scale of a few acoustic wavelengths, these double modes constitute an important example where the bulk-driven streaming is significant.

We have shown in Eq. (8) that the appearance of bulkdriven streaming in closed microcavities is directly related to the amount of rotation (acoustic angular momentum density) of the underlying acoustic fields. Whereas there is no rotation in a single mode, we have demonstrated in Secs. IV B and $\mathrm{V}$ that the double modes can be rotating not only due to an externally controlled rotating actuation, but, remarkably, also due to a nonrotating actuation coupled with a weak asymmetry in the geometry of the cavity. Furthermore, in Sec. V we have derived analytical expressions for the optimal aspect ratios that maximize the bulk-driven streaming in a nearly square cavity with a nonrotating actuation. We found that the bulk-driven acoustic streaming is sensitive to small deviations between the cavity side lengths of only $0.1 \%$.

We have validated the theory by direct numerical simulation in Sec. VI of the double modes $200+020,400+040$, and $600+060$ in a nearly square cavity with side lengths $L_{x} \approx 2000 \mu \mathrm{m}$ and $L_{y} \approx 2000 \mu \mathrm{m}$ and height $L_{z}=200 \mu \mathrm{m}$, similar to the device investigated experimentally by Hagsäter et al. [39]. Using the analytically known optimal conditions (29), for which the bulk-driven acoustic streaming is maximized, we have avoided the otherwise required timeconsuming parametric sweeping in geometry and frequency to locate these optimal conditions. The numerical results shown in Figs. 4-6 agree with the analytical expressions with a relative accuracy better than $1 \%$.

In Fig. 7, the 3D simulation of the acoustic streaming of the double mode $600+060$ is seen to reproduce the horizontally rotating $6 \times 6$ streaming-roll pattern observed by Hagsäter et al. In Fig. 8 we showed that this streaming pattern at the higher frequency $2.24 \mathrm{MHz}$ is dominated by the bulkdriven streaming, whereas the streaming of the double mode $200+020$ at the lower frequency $0.75 \mathrm{MHz}$ is dominated by the boundary-driven streaming. This frequency-dependent crossover occurs because the body force $\boldsymbol{f}_{\mathrm{ac}}$ increases as frequency to the power two, whereas the slip velocity $\boldsymbol{v}_{2}^{\text {slip }}$ depends only weakly on frequency [see Eq. (7)]. An analytical expression for computing the crossover for the double mode $l 00+0 l 0$ is given in Eq. (39).

Finally, in agreement with Eckart [29], we have shown in Eq. (37) that the bulk-driven acoustic streaming increases with the ratio $\frac{\eta_{\mathrm{fl}}^{\mathrm{b}}}{\eta_{\mathrm{fl}}}$ between the bulk and the dynamic viscosity. This dependency on $\eta_{\mathrm{fl}}^{\mathrm{b}}$ was studied numerically in Sec. VIC 6 by exchanging the water in the microcavity with pyridine. As shown in Fig. 9, we found that the bulk-driven streaming for pyridine was enhanced by a factor $\simeq 21$ relative to water. This prediction obviously calls for experimental validation.

In this paper we have pointed out the significance of the bulk-driven acoustic streaming in resonating acoustic cavities even when the geometry length scale is comparable to a few half-wavelengths. The fundamental requirement for obtaining this effect is the existence of two overlapping resonance modes excited simultaneously at the same frequency. This requirement is easily fulfilled in integrable geometries in $3 \mathrm{D}$, such as rectangular, cylindrical, and spherical cavities, or in $2 \mathrm{D}$, such as rectangular and circular cross sections of long, straight capillary channels, all of which are frequently encountered in experiments reported in the literature. The insight provided by our analysis is therefore relevant and important for acoustofluidics in general, both for fundamental studies and for technological applications.

\section{APPENDIX A: ACOUSTIC PRESSURE IN A RECTANGULAR BOX WITH BOUNDARY LAYERS}

In this Appendix, we derive the solution for the acoustic pressure $p_{1}$ with boundary layers in a rectangular-box-shaped cavity $\Omega$ for which $0 \leqslant x \leqslant L_{x}, 0 \leqslant y \leqslant L_{y}$, and $0 \leqslant z \leqslant L_{z}$. The governing equation and boundary conditions for $p_{1}$ are given in Eq. (3). We consider first an actuation $U_{1 \perp}(x, y)$ which is only nonzero at the bottom boundary $z=0$. The solution for a general actuation on all six boundaries is then finally constructed by superposition. Using separation of variables to solve the Helmholtz equation (3a) gives $p_{1}$ in the following form:

$$
\begin{aligned}
p_{1} & =X(x) Y(y) Z(z), \quad k_{\mathrm{c}}^{2}=k_{x}^{2}+k_{y}^{2}+k_{z}^{2}, \\
X(x) & =C_{x} \cos \left(k_{x} x\right)+S_{x} \sin \left(k_{x} x\right), \\
Y(y) & =C_{y} \cos \left(k_{y} y\right)+S_{y} \sin \left(k_{y} y\right), \\
Z(z) & =C_{z} \cos \left(k_{z} z\right)+S_{z} \sin \left(k_{z} z\right) .
\end{aligned}
$$

Inserting this form into boundary condition ( $3 b)$ gives

$$
\begin{gathered}
{\left[\partial_{\perp}+\alpha_{\perp}\right] p_{1}=\frac{k_{0}^{2} U_{1 \perp}}{\kappa_{\mathrm{fl}}},} \\
\alpha_{\perp}=\alpha_{\perp}\left(k_{\perp}\right)=\frac{1+i}{2} \delta\left(k_{\mathrm{c}}^{2}-k_{\perp}^{2}\right), \quad \perp=x, y, z,
\end{gathered}
$$

where $\perp$ is the inward direction perpendicular to the boundary, and the quantity $\alpha_{\perp}$ is the boundary-layer correction to the inward-normal derivative of $p_{1}$. Note that $\partial_{\perp}$ has a sign change at two opposite boundaries, say $x=0$ and $L_{x}$, for which $\partial_{\perp}=\partial_{x}$ and $-\partial_{x}$, respectively, whereas $\alpha_{\perp}\left(k_{\perp}\right)$ depends on $k_{\perp}^{2}=k_{x}^{2}$, without a sign change at opposite boundaries. We assume in the following that the length scale introduced by $\alpha_{\perp}$ is much longer than the cavity length scale $L_{\perp}$ :

$$
\left|\alpha_{\perp}\right| L_{\perp} \ll 1, \quad \perp=x, y, z .
$$

According to Eq. (A2), the boundary conditions at the stationary boundaries at $x=0$ and $L_{x}$ are

$$
\begin{array}{lc}
\underline{x=0}: & C_{x} \alpha_{x}+S_{x} k_{x}=0, \\
\underline{x=L_{x}}: & C_{x}\left[k_{x} \sin \left(k_{x} L_{x}\right)+\alpha_{x} \cos \left(k_{x} L_{x}\right)\right] \\
& +S_{x}\left[-k_{x} \cos \left(k_{x} L_{x}\right)+\alpha_{x} \sin \left(k_{x} L_{x}\right)\right]=0,
\end{array}
$$

where $\alpha_{\perp}=\alpha_{x}$ in both equations, whereas $\partial_{\perp}=\partial_{x}$ in Eq. (A4a), while $\partial_{\perp}=-\partial_{x}$ in Eq. (A4b). Nontrivial solutions with $C_{x}$ and $S_{x}$ different from zero must satisfy the usual criterion for the equation determinant $\mathcal{D}_{x}\left(k_{x}\right)$ :

$$
\begin{aligned}
& \mathcal{D}_{x}\left(k_{x}\right)=0, \text { with } \\
& \mathcal{D}_{x}\left(k_{x}\right)=\left(k_{x}^{2}-\alpha_{x}^{2}\right) \sin \left(k_{x} L_{x}\right)+2 k_{x} \alpha_{x} \cos \left(k_{x} L_{x}\right) .
\end{aligned}
$$


We write the solutions $k_{x}=k_{x}^{l}$ to Eq. (A5a) as perturbations away from the inviscid solutions $K_{x}^{l}$,

$$
k_{x}^{l}=K_{x}^{l}+\epsilon_{x}^{l}, \quad K_{x}^{l}=\frac{l \pi}{L_{x}}, \quad l=0,1,2, \ldots,
$$

where $\epsilon_{x}^{l} L_{x} \ll 1$. By inserting this into Eq. (A5b), expanding in $\epsilon_{x}^{l}$, and writing $\alpha_{x}^{l}=\alpha_{x}\left(k_{x}^{l}\right)$, we find

$$
\mathcal{D}_{x}\left(k_{x}^{l}\right)=(-1)^{l}\left\{\left[\left(K_{x}^{l}+\epsilon_{x}^{l}\right)^{2}-\left(\alpha_{x}^{l}\right)^{2}\right] \epsilon_{x}^{l} L_{x}+2\left(K_{x}^{l}+\epsilon_{x}^{l}\right) \alpha_{x}^{l}\right\} .
$$

From assumption (A3), $\left(\alpha_{x}^{l}\right)^{2} \epsilon_{x}^{l} L_{x}=\left[\alpha_{x}^{l} L_{x}\right] \epsilon_{x}^{l} \alpha_{x}^{l}$ is much smaller than $2 \epsilon_{x}^{l} \alpha_{x}^{l}$, so it can be ignored. We then factorize out $K_{x}^{l}+\epsilon_{x}^{l}=k_{x}^{l}$ and obtain

$$
\begin{aligned}
\mathcal{D}_{x}\left(k_{x}^{l}\right) & \approx(-1)^{l} k_{x}^{l}\left\{\left(K_{x}^{l}+\epsilon_{x}^{l}\right) \epsilon_{x}^{l} L_{x}+2 \alpha_{x}^{l}\right\} \\
& =(-1)^{l} k_{x}^{l}\left\{k_{x}^{l}\left(k_{x}^{l}-K_{x}^{l}\right) L_{x}+2 \alpha_{x}^{l}\right\} .
\end{aligned}
$$

For $l=0$, we have $K_{x}^{0}=0$ and $k_{x}^{0}\left(k_{x}^{0}-K_{x}^{0}\right)=\left(k_{x}^{0}\right)^{2}$, while for $l>0$ we have $k_{x}^{l}\left(k_{x}^{l}-K_{x}^{l}\right) \approx \frac{1}{2}\left(k_{x}^{l^{x}}+K_{x}^{l}\right)\left(k_{x}^{l}-K_{x}^{l}\right)$ to first order in $\epsilon_{x}^{l}$. In either case, by introducing $L_{x}^{l}$ as

$$
L_{x}^{l}=\frac{1}{2}\left(1+\delta_{0 l}\right) L_{x},
$$

the resulting expression for $\mathcal{D}_{x}\left(k_{x}^{l}\right)$ for all $l$ is written as

$$
\mathcal{D}_{x}\left(k_{x}^{l}\right) \approx(-1)^{l} k_{x}^{l} L_{x}^{l}\left[\left(k_{x}^{l}\right)^{2}-\left(K_{x}^{l}\right)^{2}+\frac{2 \alpha_{x}^{l}}{L_{x}^{l}}\right] .
$$

The wave numbers $k_{x}^{l}$ thus fulfill the zero-determinant criterion $(\mathrm{A} 5 \mathrm{a}) \mathcal{D}_{x}\left(k_{x}^{l}\right)=0$ if

$$
\left(k_{x}^{l}\right)^{2}=\left(K_{x}^{l}\right)^{2}-\frac{2 \alpha_{x}^{l}}{L_{x}^{l}},
$$

and similarly for $\left(k_{y}^{m}\right)^{2}$ for all $l$ and $m$. From Eqs. (A1) and (A4a) with $k_{x}=k_{x}^{l}$, we obtain the corresponding eigenfunctions $X^{l}(x)$,

$$
X^{l}(x)=\cos \left(k_{x}^{l} x\right)-\frac{\alpha_{x}^{l}}{k_{x}^{l}} \sin \left(k_{x}^{l} x\right),
$$

where the prefactor $C_{x}^{l}$ is absorbed into $Z(z)$ in Eq. (A1). We examine the ratio $\frac{\alpha_{x}^{l}}{k_{x}^{l}}$ for all $l$ using Eq. (A11):

$$
\begin{aligned}
\left(\frac{\alpha_{x}^{0}}{k_{x}^{0}}\right)^{2} & =-\frac{1}{2} L_{x} \alpha_{x}^{0} \ll 1, \quad l=0, \\
\frac{\alpha_{x}^{l}}{k_{x}^{l}} & =\frac{1}{l \pi} L_{x} \alpha_{x}^{l} \ll 1, \quad l>0 .
\end{aligned}
$$

By assumption (A3), we note that even for $l=0$ this ratio is small, but not as small as for $l>0$.

By inserting $k_{x}^{l}$ from Eq. (A11) into Eq. (A12) and Taylor expanding in $\alpha_{x}^{l} / k_{x}^{l}$, we recover the usual inviscid hard-wall eigenfunctions $\cos \left(K_{x}^{l} x\right)$ plus a small correction of the order $\alpha_{x}^{l} L_{x}$, due to the boundary layers

$$
X^{l}(x) \approx \cos \left(K_{x}^{l} x\right)+\alpha_{x}^{l} L_{x}\left\{\begin{array}{l}
\frac{x}{L_{x}}\left(\frac{x}{L_{x}}-1\right), \quad l=0, \\
\frac{\sin \left(K_{x}^{l} x\right)}{K_{x}^{l} L_{x}}\left(\frac{2 x}{L_{x}}-1\right), \quad l>0 .
\end{array}\right.
$$

Note that $\left.\left(\partial_{x}+\alpha_{x}^{l}\right) X^{l}\right|_{x=0}=\left.\left(\partial_{-x}+\alpha_{x}^{l}\right) X^{l}\right|_{x=L_{x}}=0$ as required by the boundary condition (A2). Similar expressions are valid for the $y$ eigenfunctions $Y^{m}(y)$.

In general, the pressure is an infinite sum of the eigenfunctions

$$
\begin{aligned}
p_{1} & =\sum_{l, m=0}^{\infty} X^{l} Y^{m} Z^{l m}(z), \\
Z^{l m}(z) & =C_{z}^{l m} \cos \left(k_{z}^{l m} z\right)+S_{z}^{l m} \sin \left(k_{z}^{l m} z\right), \\
\left(k_{z}^{l m}\right)^{2} & =k_{\mathrm{c}}^{2}-\left(k_{x}^{l}\right)^{2}-\left(k_{y}^{m}\right)^{2},
\end{aligned}
$$

where $k_{z}^{l m}$ depends on the angular frequency $\omega$ of the actuation through $k_{\mathrm{c}}$. At $z=L_{z}$, the sets of coefficients $C_{z}^{l m}$ and $S_{z}^{l m}$ satisfy a condition similar to Eq. (A4b), which leads to

$$
S_{z}^{l m}=\frac{k_{z}^{l m} \sin \left(k_{z}^{l m} L_{z}\right)+\alpha_{z}^{l m} \cos \left(k_{z}^{l m} L_{z}\right)}{k_{z}^{l m} \cos \left(k_{z}^{l m} L_{z}\right)-\alpha_{z}^{l m} \sin \left(k_{z}^{l m} L_{z}\right)} C_{z}^{l m},
$$

where $\alpha_{z}^{l m}=\alpha_{z}\left(k_{z}^{l m}\right)$. Further, at $z=0$ we have a condition similar to Eq. (A4a) with $k_{0}^{2} \kappa_{\mathrm{fl}}^{-1} U_{1 z}(x, y)$ on the right-hand side. Combined with Eqs. (A15) and (A16), the boundary condition at $z=0$ becomes

$$
\sum_{l, m=0}^{\infty} \frac{X^{l}(x) Y^{m}(y) \mathcal{D}_{z}\left(k_{z}^{l m}\right) C_{z}^{l m}}{k_{z}^{l m} \cos \left(k_{z}^{l m} L_{z}\right)-\alpha_{z}^{l m} \sin \left(k_{z}^{l m} L_{z}\right)}=\frac{k_{0}^{2} U_{1 z}(x, y)}{\kappa_{\mathrm{fl}}},
$$

where $\mathcal{D}_{z}$ is defined similar to Eq. (A5b). To find the coefficients $C_{z}^{l m}$, we write the wall actuation function $U_{1 z}(x, y)$ as a generalized Fourier series in the functions $X^{l}(x)$ and $Y^{m}(y)$ that form a complete basis set on the interval $0 \leqslant x \leqslant L_{x}$ and $0 \leqslant y \leqslant L_{y}$ to order $\alpha_{x}^{l} L_{x}$ and $\alpha_{y}^{m} L_{y}$, respectively:

$$
\begin{aligned}
U_{1 z}(x, y) & =\sum_{l, m=0}^{\infty} \hat{U}_{1 z}^{l m} X^{l}(x) Y^{m}(y), \\
\hat{U}_{1 z}^{l m} & =\frac{\int_{0}^{L_{y}} \int_{0}^{L_{x}} U_{1 z}(x, y) X^{l}(x) Y^{m}(y) d A}{\int_{0}^{L_{y}} \int_{0}^{L_{x}}\left[X^{l}(x) Y^{m}(y)\right]^{2} d A} .
\end{aligned}
$$

Inserting the expansion (A18a) into Eq. (A17), we obtain the amplitudes $C_{z}^{l m}$ :

$$
C_{z}^{l m} \approx \frac{k_{z}^{l m} \cos \left(k_{z}^{l m} L_{z}\right)-\alpha_{z}^{l m} \sin \left(k_{z}^{l m} L_{z}\right)}{\mathcal{D}_{z}\left(k_{z}^{l m}\right)} \frac{k_{0}^{2} \hat{U}_{1 z}^{l m}}{\kappa_{\mathrm{fl}}} .
$$

Finally, using Eq. (A16) for $S_{z}^{l m}$ and Eq. (A19) for $C_{z}^{l m}$ in Eq. (A15) for $p_{1}$, yields the expression for the pressure

$$
\begin{aligned}
p_{1} & =\sum_{l m=0}^{\infty} \frac{k_{0}^{2} k_{z}^{l m} \hat{U}_{1 z}^{l m}}{\kappa_{\mathrm{fl}} \mathcal{D}_{z}\left(k_{z}^{l m}\right)} X^{l}(x) Y^{m}(y) Z^{l m}(z), \\
Z^{l m}(z) & =\cos \left[k_{z}^{l m}\left(L_{z}-z\right)\right]-\frac{\alpha_{z}^{l m}}{k_{z}^{l m}} \sin \left[k_{z}^{l m}\left(L_{z}-z\right)\right], \\
{\left[k_{z}^{l m}\left(k_{0}\right)\right]^{2} } & =\left(1+i \Gamma_{\mathrm{fl}}\right) k_{0}^{2}-\left(k_{x}^{l}\right)^{2}-\left(k_{y}^{m}\right)^{2} .
\end{aligned}
$$


The infinite sum in Eq. (A20a) is general and applies to all frequencies and actuations at the bottom boundary. For a given actuation $U_{1 z}$, this solution is largest for frequencies $\omega=c_{\mathrm{fl}} k_{0}$ where $\mathcal{D}_{z}\left(k_{z}^{l m}\right)$ is smallest, which gives the hard-wall resonances $l m n$ studied below. In real systems, the surrounding solid will have its own resonance properties and, therefore, the actuation $U_{1 z}$ will depend strongly on the frequency. In this case, the largest value of the prefactor $\frac{k_{0}^{2} k_{z}^{l m} \tilde{U}_{l z}^{l m}}{\kappa_{\mathrm{f}} \mathcal{D}_{z}\left(k_{z}^{l m}\right)}$ is not necessarily found where $\mathcal{D}_{z}\left(k_{z}^{l m}\right)$ is smallest.

\section{Single-mode resonances in a rectangular box}

Resonance occurs when $k_{0}=\frac{1}{c_{\mathrm{f}}} \omega$ equals one of the values $k_{0}^{l m n}$ that minimize $\mathcal{D}_{z}\left(k_{z}^{l m}\right)$ in Eq. (A20a). We expect these values of $k_{0}^{l m n}$ to be near the inviscid values $K_{0}^{l m n}$, and write, similar to Eq. (A6),

$$
\begin{aligned}
k_{0}^{l m n} & =K_{0}^{l m n}+\epsilon_{0}^{l m n}, \\
K_{0}^{l m n} & =\sqrt{\left(K_{x}^{l}\right)^{2}+\left(K_{y}^{m}\right)^{2}+\left(K_{z}^{n}\right)^{2}}, \\
K_{x}^{l} & =\frac{l \pi}{L_{x}}, \quad K_{y}^{m}=\frac{m \pi}{L_{y}}, \quad K_{z}^{n}=\frac{n \pi}{L_{z}},
\end{aligned}
$$

where $\epsilon_{0}^{l m n}$ should be chosen to minimize $\mathcal{D}_{z}\left(k_{z}^{l m}\right)$. The modes $l$ and $m$ in the $x$ and $y$ directions together with the resonance condition $k_{0}=k_{0}^{l m n}$ fix the wave number $k_{z}^{l m n}=k_{z}^{l m}\left(k_{0}^{l m n}\right)$ in Eq. (A20c) pertaining to the $z$ direction:

$$
\left(k_{z}^{l m n}\right)^{2}=\left(1+i \Gamma_{\mathrm{fl}}\right)\left(k_{0}^{l m n}\right)^{2}-\left(k_{x}^{l}\right)^{2}-\left(k_{y}^{m}\right)^{2} .
$$

Combining this expression with Eq. (A11) for $\left(k_{x}^{l}\right)^{2}$ and $\left(k_{y}^{m}\right)^{2}$, we find

$$
\begin{aligned}
\left(k_{z}^{l m n}\right)^{2}= & \left(K_{0}^{l m n}+\epsilon_{0}^{l m n}\right)^{2}\left(1+i \Gamma_{\mathrm{fl}}^{l m n}\right) \\
& -\left[\left(K_{x}^{l}\right)^{2}-\frac{2 \alpha_{x}^{l}}{L_{x}^{l}}\right]-\left[\left(K_{y}^{m}\right)^{2}-\frac{2 \alpha_{y}^{m}}{L_{y}^{m}}\right] \\
\approx & \left(K_{z}^{n}\right)^{2}+2 K_{0}^{l m n} \epsilon_{0}^{l m n}+i \Gamma_{\mathrm{fl}}^{l m n}\left(k_{0}^{l m n}\right)^{2}+\frac{2 \alpha_{x}^{l}}{L_{x}^{l}}+\frac{2 \alpha_{y}^{m}}{L_{y}^{m}} .
\end{aligned}
$$

To evaluate $\mathcal{D}_{z}\left(k_{z}^{l m n}\right)$ in Eq. (A20a), we note that $k_{z}^{l m n}$ is close to $K_{z}^{n}$, so we can use Eq. (A10) with $\left(k_{x}^{l}\right)^{2}-\left(K_{x}^{l}\right)^{2} \rightarrow$ $\left(k_{z}^{l m n}\right)^{2}-\left(K_{z}^{n}\right)^{2}$, which is found from Eq. (A23):

$$
\begin{aligned}
\mathcal{D}_{z}\left(k_{z}^{l m n}\right) \approx & (-1)^{n} L_{z}^{n} k_{z}^{l m n}\left[2 K_{0}^{l m n} \epsilon_{0}^{l m n}+i \Gamma_{\mathrm{fl}}^{l m n}\left(k_{0}^{l m n}\right)^{2}\right. \\
& \left.+\frac{2 \alpha_{x}^{l}}{L_{x}^{l}}+\frac{2 \alpha_{y}^{m}}{L_{y}^{m}}+\frac{2 \alpha_{z}^{n}}{L_{z}^{n}}\right] .
\end{aligned}
$$

The (real) value of $\epsilon_{0}^{l m n}$, which minimizes this expression and leads to the resonance wave number $k_{0}^{l m n}$, is then

$$
\begin{aligned}
\epsilon_{0}^{l m n} & =-\operatorname{Re}\left[\frac{\alpha_{x}^{l}}{K_{0}^{l m n} L_{x}^{l}}+\frac{\alpha_{y}^{m}}{K_{0}^{l m n} L_{y}^{m}}+\frac{\alpha_{z}^{n}}{K_{0}^{l m n} L_{z}^{n}}\right] \\
& =-\frac{1}{2} K_{0}^{l m n} \Gamma_{\mathrm{bl}}^{l m n},
\end{aligned}
$$

$$
\begin{aligned}
\Gamma_{\mathrm{bl}}^{l m n}= & \frac{1}{\left(K_{0}^{l m n}\right)^{2}} \operatorname{Re}\left[\frac{2 \alpha_{x}^{l}}{L_{x}^{l}}+\frac{2 \alpha_{y}^{m}}{L_{y}^{m}}+\frac{2 \alpha_{z}^{n}}{L_{z}^{n}}\right] \\
= & \left(\frac{K_{x}^{l}}{K_{0}^{l m n}}\right)^{2}\left(\frac{\delta}{L_{y}^{m}}+\frac{\delta}{L_{z}^{n}}\right)+\left(\frac{K_{y}^{m}}{K_{0}^{l m n}}\right)^{2}\left(\frac{\delta}{L_{z}^{n}}+\frac{\delta}{L_{x}^{l}}\right) \\
& +\left(\frac{K_{z}^{n}}{K_{0}^{l m n}}\right)^{2}\left(\frac{\delta}{L_{x}^{l}}+\frac{\delta}{L_{y}^{m}}\right),
\end{aligned}
$$

where we have inserted the expressions for $\alpha_{x}^{l}, \alpha_{y}^{m}$, and $\alpha_{z}^{n}$ defined by Eq. (A2b), and introduced the boundary-layer damping coefficients $\Gamma_{\mathrm{bl}}^{\operatorname{lm} n}$.

We are now in a position to determine the third and last wave number $k_{z}^{l m n}$, the one in the actuation direction. Using the value of $\epsilon_{0}^{l m n}$ from Eq. (A25a) in expression (A23) for $k_{z}^{l m n}$, we find

$$
\begin{aligned}
\left(k_{z}^{l m n}\right)^{2}= & \left(K_{z}^{n}\right)^{2}+i \Gamma_{\mathrm{fl}}^{l m n}\left(k_{0}^{l m n}\right)^{2} \\
& +i \operatorname{Re}\left[\frac{2 \alpha_{x}^{l}}{L_{x}^{l}}+\frac{2 \alpha_{y}^{m}}{L_{y}^{m}}\right]-\operatorname{Re}\left[\frac{2 \alpha_{z}^{n}}{L_{z}^{n}}\right] \\
= & \left(K_{z}^{n}\right)^{2}+i \Gamma_{\mathrm{fl}}^{l m n}\left(k_{0}^{l m n}\right)^{2}+i\left(K_{0}^{l m n}\right)^{2} \Gamma_{\mathrm{bl}}^{l m n}-\frac{2 \alpha_{z}^{n}}{L_{z}^{n}} \\
\approx & \left(K_{z}^{n}\right)^{2}-\frac{2 \alpha_{z}^{n}}{L_{z}^{n}}+i\left(k_{0}^{l m n}\right)^{2} \Gamma^{l m n} \\
= & \left(k_{z}^{n}\right)^{2}+i\left(k_{0}^{l m n}\right)^{2} \Gamma^{l m n},
\end{aligned}
$$

with $k_{z}^{n}$ defined similar to $k_{x}^{l}$ in Eq. (A11) and the total damping coefficient given by

$$
\Gamma^{l m n}=\Gamma_{\mathrm{bl}}^{l m n}+\Gamma_{\mathrm{fl}}^{l m n} .
$$

Note that in comparison with $k_{x}^{l}$ and $k_{y}^{m}$, the wave number $k_{z}^{l m n}$ has an additional dependency on $\Gamma^{l m n}$. The corresponding $z-$ dependent functions $Z^{l m n}(z)$ are computed to lowest order in the small parameters by inserting Eq. (A26) into Eq. (A20b):

$$
\begin{aligned}
Z^{l m n}(z) \approx & (-1)^{n}\left[Z^{n}(z)+i\left(k_{0}^{l m n} L_{z}\right)^{2} \Gamma^{l m n} Z_{\text {act }}^{n}(z)\right], \\
Z_{\text {act }}^{n}(z) & =\left\{\begin{array}{l}
-\frac{1}{2}\left(1-\frac{z}{L_{z}}\right)^{2}, \quad n=0, \\
\frac{1}{2} \frac{\sin \left(K_{z}^{n} z\right)}{K_{z}^{n} L_{z}}\left(1-\frac{z}{L_{z}}\right), \quad n>0 .
\end{array}\right.
\end{aligned}
$$

Here, $Z^{l m n}(z)$ is close to the eigenfunction $Z^{n}(z)$ for stationary boundaries, analogous to $X^{l}(x)$ and $Y^{m}(y)$ [see Eq. (A12)], but it contains an extra term, which satisfies the boundary condition at the moving actuated boundary $z=0$.

Finally, we evaluate $\mathcal{D}_{z}\left(k_{z}^{l m n}\right)$ in Eq. (A24) at resonance $k_{0}=k_{0}^{l m n}$ where $\epsilon_{0}^{l m n}$ takes the value given in Eq. (A25a),

$$
\mathcal{D}_{z}\left(k_{z}^{l m n}\right) \approx(-1)^{n} L_{z}^{n} k_{z}^{l m n} i \Gamma^{l m n}\left(k_{0}^{l m n}\right)^{2}
$$

and by inserting Eqs. (A29) and (A28) into Eq. (A20a), we find an expression for each resonance mode $p_{1}^{l m n}$ :

$$
\begin{aligned}
p_{1}^{l m n} & =\frac{\left(k_{0}^{l m n}\right)^{2} k_{z}^{l m n} \hat{U}_{1 z}^{l m}}{\kappa_{\mathrm{fl}} \mathcal{D}_{z}\left(k_{z}^{l m n}\right)} X^{l}(x) Y^{m}(y) Z^{l m n}(z) \\
& =\frac{\left(k_{0}^{l m n}\right)^{2} \hat{U}_{1 z}^{l m}}{\kappa_{\mathrm{fl}} L_{z}^{n}} X^{l}(x) Y^{m}(y)\left[\frac{Z^{n}(z)}{i\left(k_{0}^{l m n}\right)^{2} \Gamma^{l m n}}+L_{z}^{2} Z_{\mathrm{act}}^{n}(z)\right]
\end{aligned}
$$


Note that for any $l, m$, and $n$, the product term $X^{l}(x) Y^{m}(y) Z^{n}(z)$ only satisfies the boundary condition (A2a) for a stationary boundary $U_{1 \perp}=0$. The actual boundary condition is satisfied by the infinite sum (A20a) over terms $X^{l}(x) Y^{m}(y) Z_{\text {act }}^{n}(z)$ because $\partial_{z} Z_{\text {act }}^{n}=\frac{L_{z}^{n}}{\left(L_{z}\right)^{2}}$ at $z=0$ and $\partial_{z} Z_{\text {act }}^{n}=$ 0 at $z=L_{z}$.

As Eq. (A30) is only valid exactly at resonance $k_{0}=k_{0}^{l m n}$, it must be corrected to deal with frequencies $k_{0} \approx k_{0}^{l m n}$. This is done by adding $k_{0}-k_{0}^{l m n}$ to the right-hand side of Eq. (A21). By Eq. (A24), this procedure is seen to be equivalent to the substitution

$$
i\left(k_{0}^{l m n}\right)^{2} \Gamma^{l m n} \rightarrow 2 k_{0}^{l m n}\left(k_{0}-k_{0}^{l m n}\right)+i\left(k_{0}^{l m n}\right)^{2} \Gamma^{l m n}
$$

in Eq. (A30).

\section{Approximate solutions near resonance for actuation at all boundaries}

At resonance, the expression (A30) satisfies all boundary conditions to first order in $\alpha_{x}^{l} L_{x}, \alpha_{y}^{m} L_{y}$, and $\alpha_{z}^{n} L_{z}$. Ignoring these first-order corrections as well as the small $Z_{\text {act }}^{n}$ term in Eq. (A30), the eigenfunctions are approximately equal to the usual hard-wall eigenfunctions $R^{l m n}$ :

$$
R^{l m n}(x, y, z)=\cos \left(K_{x}^{l} x\right) \cos \left(K_{y}^{m} y\right) \cos \left(K_{z}^{n} z\right) .
$$

To generalize our results from an actuation acting on only the bottom boundary $z=0$ to all six boundaries, we note first that $\left.L_{z}^{n} \approx \int_{0}^{L_{z}}\left[Z^{n}(z)\right)\right]^{2} d z$, whereby Eq. (A18b) can be rewritten as

$$
\begin{aligned}
\frac{\hat{U}_{1 z}^{l m}}{L_{z}^{n}} & =\frac{\int_{0}^{L_{y}} \int_{0}^{L_{x}} U_{1 z}(x, y) X^{l}(x) Y^{m}(y) d A}{L_{z}^{n} \int_{0}^{L_{y}} \int_{0}^{L_{x}}\left[X^{l}(x) Y^{m}(y)\right]^{2} d A} \\
& \approx \frac{\int_{0}^{L_{y}} \int_{0}^{L_{x}} U_{1 z}(x, y) R^{l m n}(x, y, 0) d A}{\int_{0}^{L_{z}} \int_{0}^{L_{y}} \int_{0}^{L_{x}}\left[R^{l m n}(x, y, z)\right]^{2} d V} .
\end{aligned}
$$

Using this expression together with the substitution (A31) valid for $k_{0} \approx k_{0}^{l m n}$, we obtain the following expression for the resonance modes $l m n$ in a rectangular box $\Omega$ with viscous boundary layers and an inward normal displacement $U_{1 \perp}(\boldsymbol{r})$ specified on all six boundaries $\partial \Omega$ [Eq. (3c)]:

$$
\begin{aligned}
p_{1}^{l m n} & =P_{1}^{l m n} F^{l m n}\left(k_{0}\right) R^{l m n}(\boldsymbol{r}), \\
P_{1}^{l m n} & =\frac{\rho_{\mathrm{fl}} c_{\mathrm{fl}}^{2}}{\Gamma^{l m n}} \frac{\int_{\partial \Omega} U_{1 \perp} R^{l m n} d A}{\int_{\Omega}\left(R^{l m n}\right)^{2} d V}, \\
F^{l m n}\left(k_{0}\right) & =\frac{\frac{1}{2} k_{0}^{l m n} \Gamma^{l m n}}{\left(k_{0}-k_{0}^{l m n}\right)+i \frac{1}{2} k_{0}^{l m n} \Gamma^{l m n}} .
\end{aligned}
$$

The quality factor $Q^{l m n}$ for resonance mode $l m n$ is obtained from the linewidth of the acoustic energy density $E_{\mathrm{ac}}$ in Eq. (6), and since $E_{\mathrm{ac}} \propto\left|p_{1}^{l m n}\right|^{2} \propto\left|F^{l m n}\right|^{2}$, we find

$$
Q^{l m n} \approx \frac{1}{\Gamma^{l m n}}
$$

where $\Gamma^{l m n}$ is given in Eq. (A27).

\section{APPENDIX B: MAXIMUM ACOUSTIC ROTATION}

In the following, we evaluate the frequency-dependent factor $\mathcal{F}_{m l 0}^{\operatorname{lm} 0}$ in Eq. (17b) for rotating and nonrotating actuations of the nearly square cavity considered in Sec. V. For convenience, we first introduce the double-mode quantities $\tilde{\xi}_{l m}$ and $\phi_{l m}^{\text {act }}$,

$$
\tilde{\xi}_{l m}=\tilde{k}_{0}-\tilde{k}_{l m}^{\star}, \quad \phi_{l m}^{\mathrm{act}}=\phi_{\mathrm{act}}^{m l 0}-\phi_{\mathrm{act}}^{l m 0},
$$

where $\tilde{k}_{0}$ and $\tilde{k}_{l m}^{\star}$ are defined in Eq. (27), and $\phi_{l m}^{\text {act }}$ is the difference in the phases by which the modes $\operatorname{lm} 0$ and $m l 0$ are actuated [see Eq. (12b)]. By using these quantities and the expressions for $F^{l m 0}$ and $F^{m l 0}$ in Eq. (28), we calculate the frequency-dependency $\mathcal{F}_{m l 0}^{l m 0}$ from Eq. (17b):

$$
\begin{aligned}
\mathcal{F}_{m l 0}^{l m 0} & =\operatorname{Re}\left\{i e^{i \phi_{l m}^{\mathrm{act}}} F^{l m 0}\left[F^{m l 0}\right]^{*}\right\} \\
& =\frac{\cos \left(\phi_{l m}^{\mathrm{act}}\right) 2 \tilde{\Delta}_{l m}+\sin \left(\phi_{l m}^{\mathrm{act}}\right)\left[1+\left(2 \tilde{\xi}_{l m}\right)^{2}-\tilde{\Delta}_{l m}^{2}\right]}{\left[\left(2 \tilde{\xi}_{l m}-\tilde{\Delta}_{l m}\right)^{2}+1\right]\left[\left(2 \tilde{\xi}_{l m}+\tilde{\Delta}_{l m}\right)^{2}+1\right]} .
\end{aligned}
$$

This equation expresses the magnitude of the acoustic rotation as a function of the actuation phase difference $\phi_{l m}^{\text {act }}$, the actuation frequency $\tilde{\xi}_{l m}$, and the mode separation $\tilde{\Delta}_{l m}$, which by Eq. (26) is related to the aspect ratio $A$. In the following, we study the nonrotating actuation with $\phi_{l m}^{\text {act }}=0$ and the rotating actuation with $\phi_{l m}^{\text {act }}= \pm \frac{1}{2} \pi$, and we determine the optimal mode separation $\tilde{\Delta}_{l m}^{\text {opt }}$ and frequency $\tilde{\xi}_{l m}^{\text {opt }}$, for which $\mathcal{F}_{m l 0}^{l m 0}$ takes its largest positive or negative value $\left(\mathcal{F}_{m l 0}^{l m 0}\right)^{\text {extr }}$.

\section{Extremum values of $\mathcal{F}_{m l 0}^{l m 0}$ for a nonrotating actuation $\phi_{l m}^{\text {act }}=0$}

For a nonrotating actuation, we have $\phi_{l m}^{\text {act }}=0$, and Eq. (B2) becomes

$$
\mathcal{F}_{m l 0}^{l m 0}=\frac{2 \tilde{\Delta}_{l m}}{\left[\left(2 \tilde{\xi}_{l m}-\tilde{\Delta}_{l m}\right)^{2}+1\right]\left[\left(2 \tilde{\xi}_{l m}+\tilde{\Delta}_{l m}\right)^{2}+1\right]} .
$$

The extremum values of this expressions are

$$
\left(\mathcal{F}_{m l 0}^{l m 0}\right)^{\text {extr }}= \begin{cases}\frac{2 \tilde{\Delta}_{l m}}{\left(\tilde{\Delta}_{l m}^{2}+1\right)^{2}}, & \left|\tilde{\Delta}_{l m}\right|<1, \\ \frac{1}{2 \tilde{\Delta}_{l m}}, & \left|\tilde{\Delta}_{l m}\right|>1,\end{cases}
$$

which are obtained at the frequencies $\tilde{\xi}_{l m}^{\text {extr }}$ given by

$$
\tilde{\xi}_{l m}^{\text {extr }}= \begin{cases}0, & \left|\tilde{\Delta}_{l m}\right|<1 \\ \pm \frac{1}{2} \sqrt{\tilde{\Delta}_{l m}^{2}-1,} & \left|\tilde{\Delta}_{l m}\right|>1\end{cases}
$$

In Fig. 6 ("nonrotating act."), we plot the value $\left(\mathcal{F}_{m l 0}^{l m 0}\right)^{\text {extr }}$ from this expression as a function of $\tilde{\Delta}_{l m}$. The optimal value $\left(\mathcal{F}_{m l 0}^{l m 0}\right)^{\mathrm{opt}}$ of $\left(\mathcal{F}_{m l 0}^{l m 0}\right)^{\mathrm{extr}}$ is found at the optimal mode separation $\tilde{\Delta}_{l m}^{\mathrm{opt}}$, and optimal rescaled frequency $\tilde{\xi}_{l m}^{\mathrm{opt}}$ :

$$
\tilde{\xi}_{l m}^{\mathrm{opt}}=0, \quad \tilde{\Delta}_{l m}^{\mathrm{opt}}= \pm \frac{1}{\sqrt{3}}, \quad\left(\mathcal{F}_{m l 0}^{l m 0}\right)^{\mathrm{opt}}= \pm \frac{3 \sqrt{3}}{8} .
$$

2. Extremum values of $\mathcal{F}_{m l 0}^{l m 0}$ for a rotating actuation $\phi_{l m}^{\text {act }}= \pm \frac{1}{2} \pi$ For an externally controlled rotating actuation, we have $\phi_{l m}^{\text {act }}=$ $\pm \frac{1}{2} \pi$ and Eq. (B2) becomes

$$
\begin{aligned}
\mathcal{F}_{m l 0}^{l m 0} & =\frac{\left[1+\left(2 \tilde{\xi}_{l m}\right)^{2}-\tilde{\Delta}_{l m}^{2}\right] \operatorname{sgn}_{\phi}^{l m}}{\left[\left(2 \tilde{\xi}_{l m}-\tilde{\Delta}_{l m}\right)^{2}+1\right]\left[\left(2 \tilde{\xi}_{l m}+\tilde{\Delta}_{l m}\right)^{2}+1\right]}, \\
\operatorname{sgn}_{\phi}^{l m} & =\operatorname{sign}\left(\phi_{l m}^{\mathrm{act}}\right) .
\end{aligned}
$$


This expression is more complicated than Eq. (B3) since it only has one extremum for a sufficiently small mode separation $\tilde{\Delta}_{l m}$, namely, where the angular momentum and the actuation are corotating, $\operatorname{sign}\left(\mathcal{F}_{m l 0}^{\operatorname{lm} 0}\right)=\operatorname{sgn}_{\phi}^{l m}$. For larger values of $\tilde{\Delta}_{l m}$, the frequency can be tuned to two different extremum values, namely, both the corotating and counterrotating cases, $\operatorname{sign}\left(\mathcal{F}_{m l 0}^{\operatorname{lm} 0}\right)= \pm \operatorname{sgn}_{\phi}^{l m}$. We therefore identify two branches of extremum values. The corotating branch $\left(\mathcal{F}_{m l 0}^{\operatorname{lm} 0}\right)_{\mathrm{co}}^{\text {extr }}$ with subscript "co" and the counter-rotating branch $\left(\mathcal{F}_{m l 0}^{l m 0}\right)_{\text {cntr }}^{\text {extr }}$ with subscript "cntr."

For the corotating branch, $\left(\mathcal{F}_{m l 0}^{l m 0}\right)_{\mathrm{co}}^{\text {extr }}$ is

$$
\left(\mathcal{F}_{m l 0}^{l m 0}\right)_{\mathrm{co}}^{\operatorname{extr}}=\operatorname{sgn}_{\phi}^{l m} \begin{cases}\frac{1-\tilde{\Delta}_{l m}^{2}}{\left(\tilde{\Delta}_{l m}^{2}+1\right)^{2}}, & \left|\tilde{\Delta}_{l m}\right| \leqslant \sqrt{2}-1, \\ \frac{1}{4\left|\tilde{\Delta}_{l m}\right|}, & \left|\tilde{\Delta}_{l m}\right| \geqslant \sqrt{2}-1,\end{cases}
$$

found at the frequencies $\tilde{\xi}_{l m}=\tilde{\xi}_{l m, \mathrm{co}}^{\text {extr }}$ :

$$
\tilde{\xi}_{l m, \mathrm{co}}^{\mathrm{extr}}= \begin{cases}0, & \left|\tilde{\Delta}_{l m}\right| \leqslant \sqrt{2}-1 \\ \pm \frac{1}{2} \sqrt{\tilde{\Delta}_{l m}^{2}+2 \tilde{\Delta}_{l m}-1,} & \left|\tilde{\Delta}_{l m}\right| \geqslant \sqrt{2}-1\end{cases}
$$

For the counter-rotating branch, $\left(\mathcal{F}_{m l 0}^{l m 0}\right)_{\text {cntr }}^{\text {extr }}$ is

$$
\left(\mathcal{F}_{m l 0}^{l m 0}\right)_{\mathrm{cntr}}^{\mathrm{extr}}=-\operatorname{sgn}_{\phi}^{l m} \begin{cases}\frac{\tilde{\Delta}_{l m}^{2}-1}{\left(\tilde{\Delta}_{l m}^{2}+1\right)^{2}}, & 1 \leqslant\left|\tilde{\Delta}_{l m}\right| \leqslant \sqrt{2}+1, \\ \frac{1}{4\left|\tilde{\Delta}_{l m}\right|}, & \left|\tilde{\Delta}_{l m}\right| \geqslant \sqrt{2}+1,\end{cases}
$$

found at the frequencies $\tilde{\xi}_{l m}=\tilde{\xi}_{l m, \mathrm{cntr}}^{\mathrm{extr}}$ :

$$
\tilde{\xi}_{l m, \mathrm{cntr}}^{\mathrm{extr}}= \begin{cases}0, & 1 \leqslant\left|\tilde{\Delta}_{l m}\right| \leqslant \sqrt{2}+1 \\ \pm \frac{1}{2} \sqrt{\tilde{\Delta}_{l m}^{2}-2 \tilde{\Delta}_{l m}-1,} & \left|\tilde{\Delta}_{l m}\right| \geqslant \sqrt{2}+1\end{cases}
$$

In Fig. 6 (rotating act.), we plot for $\phi_{l m}^{\text {act }}=+\frac{1}{2} \pi$ both the corotating and counter-rotating branches $\left(\mathcal{F}_{m l 0}^{\operatorname{lm} 0}\right)_{\mathrm{co}}^{\text {extr }}$ and $\left(\mathcal{F}_{m l 0}^{l m 0}\right)_{\text {cntr }}^{\text {extr }}$.

From Eqs. (B7a) and (B8a), we find that the largest value $\left(\mathcal{F}_{m l 0}^{\operatorname{lm} 0}\right)^{\mathrm{opt}}$ of $\left(\mathcal{F}_{m l 0}^{\operatorname{lm} 0}\right)$ is the corotating double mode found at the optimal mode separation $\tilde{\Delta}_{l m}^{\text {opt }}$, and the rescaled frequency $\tilde{\xi}_{l m}^{\text {opt }}$ :

$$
\tilde{\xi}_{l m}^{\mathrm{opt}}=0, \quad \tilde{\Delta}_{l m}^{\mathrm{opt}}=0, \quad\left(\mathcal{F}_{m l 0}^{l m 0}\right)^{\mathrm{opt}}=\operatorname{sgn}_{\phi}^{l m} .
$$

We see that in contrast to the nonrotating actuation (B5), the rotating actuation optimizes the acoustic rotation for zeromode separation $\tilde{\Delta}_{l m}^{\mathrm{opt}}=0$.

\section{APPENDIX C: ROTATING ACTUATION $G_{16}$ OF THE DOUBLE MODE $200+020$}

In the simulation shown in Figs. 5(d)-5(f) of Sec. VIC 2 we excite the double mode $200+020$ by using the rotating actuation

$$
u_{1 z}=u_{1 z}^{0} G_{16}(x, y), \quad 0<x<L_{x}, 0<y<L_{y} .
$$

Here, $G_{16}(x, y)$ is constructed as the sum of 16 narrow Gaussians $G_{1}^{\odot}$ as follows. The primary narrow Gaussian $G_{1}^{\odot}(x, y)$ is centered around $\left(\frac{1}{8} L_{x}, \frac{1}{8} L_{y}\right)$ in the lower left corner of the domain:

$$
G_{1}^{\odot}(x, y)=\exp \left[-\frac{\left(x-\frac{L_{x}}{8}\right)^{2}}{\left(\frac{L_{x}}{16}\right)^{2}}-\frac{\left(y-\frac{L_{y}}{8}\right)^{2}}{\left(\frac{L_{y}}{16}\right)^{2}}\right] .
$$

Four versions of $G_{1}^{\odot}$, centered at $\left(\frac{2 \pm 1}{8} L_{x}, \frac{2 \pm 1}{8} L_{y}\right)$ and multiplied by specific phase factors in order to create a positive rotation direction in the $x-y$ plane, are added to form a quadruple $G_{4}$ centered around $\left(\frac{1}{4} L_{x}, \frac{1}{4} L_{y}\right)$ :

$$
\begin{aligned}
G_{4}(x, y)= & e^{-i \frac{0 \pi}{2}} G_{1}^{\odot}(x, y)+e^{-i \frac{1 \pi}{2}} G_{1}^{\odot}\left(x, y-\frac{1}{4} L_{y}\right) \\
& +e^{-i \frac{2 \pi}{2}} G_{1}^{\odot}\left(x-\frac{1}{4} L_{x}, y-\frac{1}{4} L_{y}\right) \\
& +e^{-i \frac{3 \pi}{2}} G_{1}^{\odot}\left(x-\frac{1}{4} L_{x}, y\right) .
\end{aligned}
$$

Finally, by mirroring the quadruple $G_{4}(x, y)$ across the center lines $x=\frac{1}{2} L_{x}$ and $y=\frac{1}{2} L_{y}, G_{16}$ is formed by adding the four resulting quadruples

$$
\begin{aligned}
G_{16}(x, y)= & G_{4}(x, y)+G_{4}\left(L_{x}-x, y\right) \\
& +G_{4}\left(L_{x}-x, L_{y}-y\right)+G_{4}\left(x, L_{y}-y\right) .
\end{aligned}
$$

To obtain the complex pressure amplitudes $P_{1}^{200}$ and $P_{1}^{020}$ resulting from $G_{16}$, we first evaluate the integral in the denominator of Eq. (34), where $G_{1}$ is substituted by $G_{16}$ :

$$
\begin{aligned}
& \int_{0}^{L_{x}} \int_{0}^{L_{y}} G_{16}(x, y) R^{200} \frac{d x}{L_{x}} \frac{d y}{L_{y}}=0.0942 \times e^{i 0.250 \pi} \\
& \int_{0}^{L_{x}} \int_{0}^{L_{y}} G_{16}(x, y) R^{020} \frac{d x}{L_{x}} \frac{d y}{L_{y}}=0.0942 \times e^{-i 0.250 \pi} .
\end{aligned}
$$

We then use Eq. (34) to compute the desired amplitudes

$$
\begin{aligned}
& P_{1}^{200}=\frac{2 u_{1 z}^{0}}{L_{z}\left(\Gamma_{\mathrm{fl}}^{200}+\Gamma_{\mathrm{bl}}^{200}\right) \kappa_{\mathrm{fl}}} 0.0942 \times e^{i 0.250 \pi}, \\
& P_{1}^{020}=\frac{2 u_{1 z}^{0}}{L_{z}\left(\Gamma_{\mathrm{fl}}^{200}+\Gamma_{\mathrm{bl}}^{200}\right) \kappa_{\mathrm{fl}}} 0.0942 \times e^{-i 0.250 \pi} .
\end{aligned}
$$

We see that the actuation in Eq. (C1a) excites the two modes 200 and 020 with a phase difference of

$$
\phi_{\mathrm{act}}^{020}-\phi_{\mathrm{act}}^{200}=0.500 \pi .
$$

Finally, we use Eq. (C3) to choose the amplitude $u_{1 z}^{0}$ of the actuation such that $\left|P_{1}^{200}\right|=\left|P_{1}^{020}\right|=1 \mathrm{MPa}$,

$$
u_{1 z}^{0}=\frac{L_{z}\left(\Gamma_{\mathrm{fl}}^{200}+\Gamma_{\mathrm{bl}}^{200}\right) \kappa_{\mathrm{fl}}\left|P_{1}^{200}\right|}{2 \times 0.0942}=1.614 \mathrm{~nm},
$$

where we used the parameters for water given in Table II.

\section{APPENDIX D: APPROXIMATE SOLUTION FOR THE BULK-DRIVEN STREAMING FROM DOUBLE MODES $l 00+0 l 0$}

In this Appendix, we calculate an approximate expression for the bulk-driven acoustic streaming velocity $\left(\boldsymbol{v}_{2}^{\mathrm{blk}}\right)_{0 l 0}^{l 00}=$ $\left(\boldsymbol{v}_{2}^{\mathrm{blk}}\right)_{l 0}$ driven by the acoustic body force $\boldsymbol{f}_{\mathrm{ac}}=\boldsymbol{f}_{0 l 0}^{l 00}=\boldsymbol{f}_{l 0}$ resulting from the combination of the two perpendicular, horizontal modes $l 00$ and $0 l 0$ in a square cavity. As in Eq. (24), the double subscript $l 0$ refers to the double mode $l 00+0 l 0$. The 
bulk-driven streaming velocity ( $\left.\boldsymbol{v}_{2}^{\mathrm{blk}}\right)_{l 0}$ satisfies an equation similar to Eq. (7) except for having no-slip at the boundaries:

$$
\begin{aligned}
0 & =\nabla \cdot\left(\boldsymbol{v}_{2}^{\mathrm{blk}}\right)_{l 0}, \quad \boldsymbol{r} \in \Omega, \\
\nabla\left(p_{2}^{\mathrm{blk}}\right)_{l 0} & =\eta_{\mathrm{fl}} \nabla^{2}\left(\boldsymbol{v}_{2}^{\mathrm{blk}}\right)_{l 0}+\frac{\Gamma_{\mathrm{fl}} \omega}{c_{\mathrm{fl}}^{2}} \boldsymbol{S}_{l 0}, \quad \boldsymbol{r} \in \Omega, \\
\left(\boldsymbol{v}_{2}^{\mathrm{blk}}\right)_{l 0} & =\mathbf{0}, \quad \boldsymbol{r} \in \partial \Omega .
\end{aligned}
$$

Here, $\boldsymbol{S}_{l 0}$ is defined in Eq. (18a) combined with Eq. (24) for $\mathcal{F}_{l 0}\left(k_{0}\right)$, Eq. (17c) for $\mathcal{K}_{l 0}(\boldsymbol{r})$, and Eq. (9a) for $R_{l 0}(\boldsymbol{r})$ :

$$
\begin{array}{rlr}
\boldsymbol{S}_{l 0}(\boldsymbol{r}) & =\frac{1}{2} \frac{\left|P_{1}^{l 00}\right|\left|P_{1}^{0 l 0}\right|}{\rho_{\mathrm{fl}} \omega} \mathcal{F}_{l 0}\left(k_{0}\right) \mathcal{K}_{l 0}(\boldsymbol{r}), \\
\mathcal{F}_{l 0}\left(k_{0}\right) & =\operatorname{Re}\left\{i\left[F^{l 00}\left(k_{0}\right)\right]\left[F^{0 l 0}\left(k_{0}\right)\right]^{*}\right\}, \\
\mathcal{K}_{l 0}(\boldsymbol{r}) & =k_{l 0}^{\star}\left[\sin \left(k_{l 0}^{\star} x\right) \cos \left(k_{l 0}^{\star} y\right) \boldsymbol{e}_{x}-\cos \left(k_{l 0}^{\star} x\right) \sin \left(k_{l 0}^{\star} y\right) \boldsymbol{e}_{y}\right] .
\end{array}
$$

The impact on the bulk-driven streaming from the no-slip condition at the side walls will decrease exponentially with the length scale $w$ away from the side walls, where $w$ is the minimum shear length scale, i.e., either the vertical length scale $L_{z}$ or the horizontal length scale $\left(k_{l 0}^{\star}\right)^{-1}$,

$$
w=\min \left\{L_{z},\left(k_{l 0}^{\star}\right)^{-1}\right\} .
$$

For the narrow channel considered in Sec. VI where $L_{z} / L^{\star}=$ 0.1 , we have $w / L^{\star}=0.1$ for $l=2, w / L^{\star}=\frac{1}{4 \pi}=0.08$ for $l=4$, and $w / L^{\star}=\frac{1}{6 \pi}=0.05$ for $l=6$, and therefore the vertical side walls can be ignored in the majority of the cavity for these three double modes.

To compute the acoustic streaming velocity, we ignore the side walls and assume that $\left(\boldsymbol{v}_{2}^{\mathrm{blk}}\right)_{l 0}$ is proportional to the $x y$-dependent acoustic energy flux density $\boldsymbol{S}_{l 0}(x, y)$ and some $z$-dependent function $\zeta(z)$ to be found:

$$
\left(\boldsymbol{v}_{2}^{\mathrm{blk}}\right)_{l 0}=\kappa_{\mathrm{fl}} \zeta(z) \boldsymbol{S}_{l 0}(x, y) .
$$

By using $\boldsymbol{S}_{l 0}$ from Eq. (D2a), we find the three identities

$$
\begin{aligned}
\nabla \cdot \boldsymbol{S}_{l 0} & =0, \\
\nabla^{2} \boldsymbol{S}_{l 0} & =-2\left(k_{l 0}^{\star}\right)^{2} \boldsymbol{S}_{l 0}, \\
\nabla \times \boldsymbol{S}_{l 0} & =\frac{\left|P_{1}^{l 00}\right|\left|P_{1}^{0 l 0}\right| \mathcal{F}_{l 0}}{\rho_{\mathrm{fl}} \omega}\left(k_{l 0}^{\star}\right)^{2} \sin \left(k_{l 0}^{\star} x\right) \sin \left(k_{l 0}^{\star} y\right) \boldsymbol{e}_{z} .
\end{aligned}
$$

Consequently, by Eq. (D5a), the ansatz (D4) satisfies the continuity equation (D1a). Moreover, inserting Eq. (D4) into Eq. (D1b) using $\Gamma_{\mathrm{fl}}=\left(\frac{4}{3}+\frac{\eta_{\mathrm{f}}^{\mathrm{b}}}{\eta_{\mathrm{f}}}\right) \eta_{\mathrm{ff}} \kappa_{\mathrm{fl}} \omega$ from Eqs. (4) and (D5b) we find

$$
\nabla\left(p_{2}^{\mathrm{blk}}\right)_{l 0}=\eta_{\mathrm{f}} \kappa_{\mathrm{ff}}\left[\frac{\zeta^{\prime \prime}(z)}{\left(\sqrt{2} k_{l 0}^{\star}\right)^{2}}-\zeta(z)+\frac{1}{2}\left(\frac{4}{3}+\frac{\eta_{\mathrm{fl}}^{\mathrm{b}}}{\eta_{\mathrm{fl}}}\right)\right] \boldsymbol{S}_{l 0} .
$$

Since $\boldsymbol{S}_{l 0}(x, y)$ has no $z$ component, $\left(p_{2}^{\mathrm{blk}}\right)_{l 0}$ on the left-hand side is independent of $z$. Therefore, the square bracket on the right-hand side must be a constant. This constant must be zero since otherwise taking the curl of Eq. (D6) would lead to $\mathbf{0}=\boldsymbol{\nabla} \times \boldsymbol{S}_{l 0}$ in conflict with Eq. (D5c). The condition of a vanishing square bracket in Eq. (D6) and the no-slip boundary conditions $\zeta(0)=\zeta\left(L_{z}\right)=0$ lead to the following expression for $\zeta(z)$ :

$$
\zeta(z)=\frac{1}{2}\left(\frac{4}{3}+\frac{\eta_{\mathrm{fl}}^{\mathrm{b}}}{\eta_{\mathrm{fl}}}\right)\left[1-\frac{\cosh \left(\sqrt{2} k_{l 0}^{\star}\left(z-\frac{L_{z}}{2}\right)\right)}{\cosh \left(\sqrt{2} k_{l 0}^{\star} \frac{L_{z}}{2}\right)}\right] .
$$

The solution for $\left(\boldsymbol{v}_{2}^{\text {blk }}\right)_{l 0}$ is then obtained by inserting Eqs. (D2) and (D7) in Eq. (D4).
[1] F. Petersson, L. Åberg, A. M. Swärd-Nilsson, and T. Laurell, Free flow acoustophoresis: Microfluidic-based mode of particle and cell separation, Anal. Chem. 79, 5117 (2007).

[2] O. Manneberg, B. Vanherberghen, J. Svennebring, H. M. Hertz, B. Önfelt, and M. Wiklund, A three-dimensional ultrasonic cage for characterization of individual cells, Appl. Phys. Lett. 93, 063901 (2008).

[3] X. Ding, Z. Peng, S.-C. S. Lin, M. Geri, S. Li, P. Li, Y. Chen, M. Dao, S. Suresh, and T. J. Huang, Cell separation using tiltedangle standing surface acoustic waves, Proc. Natl. Acad. Sci. USA 111, 12992 (2014).

[4] B. Hammarström, T. Laurell, and J. Nilsson, Seed particle enabled acoustic trapping of bacteria and nanoparticles in continuous flow systems, Lab Chip 12, 4296 (2012).

[5] M. Evander, O. Gidlof, B. Olde, D. Erlinge, and T. Laurell, Non-contact acoustic capture of microparticles from small plasma volumes, Lab Chip 15, 2588 (2015).

[6] D. J. Collins, C. Devendran, Z. Ma, J. W. Ng, A. Neild, and Y. Ai, Acoustic tweezers via sub-time-of-flight regime surface acoustic waves, Sci. Adv. 2, e1600089 (2016).

[7] H. G. Lim, Y. Li, M.-Y. Lin, C. Yoon, C. Lee, H. Jung, R. H. Chow, and K. K. Shung, Calibration of trapping force on cell-size objects from ultrahigh-frequency single-beam acoustic tweezer, IEEE T. Ultrason. Ferr. 63, 1988 (2016).

[8] D. Baresch, J.-L. Thomas, and R. Marchiano, Observation of a Single-Beam Gradient Force Acoustical Trap for Elastic Particles: Acoustical Tweezers, Phys. Rev. Lett. 116, 024301 (2016).

[9] P. Augustsson, C. Magnusson, M. Nordin, H. Lilja, and T. Laurell, Microfluidic, label-free enrichment of prostate cancer cells in blood based on acoustophoresis, Anal. Chem. 84, 7954 (2012).

[10] M. Antfolk, C. Magnusson, P. Augustsson, H. Lilja, and T. Laurell, Acoustofluidic, label-free separation and simultaneous concentration of rare tumor cells from white blood cells, Anal. Chem. 87, 9322 (2015).

[11] M. Antfolk, P. B. Muller, P. Augustsson, H. Bruus, and T. Laurell, Focusing of sub-micrometer particles and bacteria enabled by two-dimensional acoustophoresis, Lab Chip 14, 2791 (2014).

[12] S. Li, F. Ma, H. Bachman, C. E. Cameron, X. Zeng, and T. J. Huang, Acoustofluidic bacteria separation, J. Micromech. Microeng. 27, 015031 (2017).

[13] P. Augustsson, J. T. Karlsen, H.-W. Su, H. Bruus, and J. Voldman, Iso-acoustic focusing of cells for size-insensitive 
acousto-mechanical phenotyping, Nat. Commun. 7, 11556 (2016).

[14] L. V. King, On the acoustic radiation pressure on spheres, Proc. R. Soc. London, Ser. A 147, 212 (1934).

[15] K. Yosioka and Y. Kawasima, Acoustic radiation pressure on a compressible sphere, Acustica 5, 167 (1955).

[16] A. A. Doinikov, Acoustic radiation force on a spherical particle in a viscous heat-conducting fluid.1. general formula, J. Acoust. Soc. Am. 101, 713 (1997).

[17] M. Settnes and H. Bruus, Forces acting on a small particle in an acoustical field in a viscous fluid, Phys. Rev. E 85, 016327 (2012).

[18] J. T. Karlsen and H. Bruus, Forces acting on a small particle in an acoustical field in a thermoviscous fluid, Phys. Rev. E 92, 043010 (2015).

[19] L. Rayleigh, On the circulation of air observed in Kundt's tubes, and on some allied acoustical problems, Philos. Trans. R. Soc. London 175, 1 (1884).

[20] H. Schlichting, Berechnung ebener periodischer grenzeschichtströmungen, Phys. Z. 33, 327 (1932).

[21] P. Westervelt, The theory of steady rotational flow generated by a sound field, J. Acoust. Soc. Am. 25, 60 (1953).

[22] W. L. Nyborg, Acoustic streaming near a boundary, J. Acoust. Soc. Am. 30, 329 (1958).

[23] P. B. Muller, M. Rossi, A. G. Marin, R. Barnkob, P. Augustsson, T. Laurell, C. J. Kähler, and H. Bruus, Ultrasound-induced acoustophoretic motion of microparticles in three dimensions, Phys. Rev. E 88, 023006 (2013).

[24] P. B. Muller, R. Barnkob, M. J. H. Jensen, and H. Bruus, A numerical study of microparticle acoustophoresis driven by acoustic radiation forces and streaming-induced drag forces, Lab Chip 12, 4617 (2012).

[25] R. Barnkob, P. Augustsson, T. Laurell, and H. Bruus, Acoustic radiation- and streaming-induced microparticle velocities determined by microparticle image velocimetry in an ultrasound symmetry plane, Phys. Rev. E 86, 056307 (2012).

[26] C. Lee and T. Wang, Near-boundary streaming around a small sphere due to 2 orthogonal standing waves, J. Acoust. Soc. Am. 85, 1081 (1989).

[27] J. Vanneste and O. Bühler, Streaming by leaky surface acoustic waves, Proc. R. Soc. A 467, 1779 (2011).

[28] J. S. Bach and H. Bruus, Theory of pressure acoustics with viscous boundary layers and streaming in curved elastic cavities, J. Acoust. Soc. Am. 144, 766 (2018).

[29] C. Eckart, Vortices and streams caused by sound waves, Phys. Rev. 73, 68 (1948).

[30] A. Riaud, M. Baudoin, O. Bou Matar, J.-L. Thomas, and P. Brunet, On the influence of viscosity and caustics on acoustic streaming in sessile droplets: An experimental and a numerical study with a cost-effective method, J. Fluid Mech. 821, 384 (2017).

[31] F. Busse and T. Wang, Torque generated by orthogonal acousticwaves theory, J. Acoust. Soc. Am. 69, 1634 (1981).

[32] S. B. Q. Tran, P. Marmottant, and P. Thibault, Fast acoustic tweezers for the two-dimensional manipulation of individual particles in microfluidic channels, Appl. Phys. Lett. 101, 114103 (2012).

[33] J. Dual, P. Hahn, I. Leibacher, D. Möller, T. Schwarz, and J. Wang, Acoustofluidics 19: Ultrasonic microrobotics in cavities: Devices and numerical simulation, Lab Chip 12, 4010 (2012).

[34] C. R. P. Courtney, B. W. Drinkwater, C. E. M. Demore, S. Cochran, A. Grinenko, and P. D. Wilcox, Dexterous manipulation of microparticles using bessel-function acoustic pressure fields, Appl. Phys. Lett. 102, 123508 (2013).

[35] Z. Mao, P. Li, M. Wu, H. Bachman, N. Mesyngier, X. Guo, S. Liu, F. Costanzo, and T. J. Huang, Enriching nanoparticles via acoustofluidics, ACS Nano 11, 603 (2017).

[36] D. Baresch, J.-L. Thomas, and R. Marchiano, Orbital Angular Momentum Transfer to Stably Trapped Elastic Particles in Acoustical Vortex Beams, Phys. Rev. Lett. 121, 074301 (2018).

[37] P. L. Marston, Axial radiation force of a bessel beam on a sphere and direction reversal of the force, J. Acoust. Soc. Am. 120, 3518 (2006).

[38] A. Marzo, S. A. Seah, B. W. Drinkwater, D. R. Sahoo, B. Long, and S. Subramanian, Holographic acoustic elements for manipulation of levitated objects, Nat. Commun. 6, 8661 (2015).

[39] S. M. Hagsäter, T. G. Jensen, H. Bruus, and J. P. Kutter, Acoustic resonances in microfluidic chips: Full-image microPIV experiments and numerical simulations, Lab Chip 7, 1336 (2007).

[40] J. T. Karlsen, W. Qiu, P. Augustsson, and H. Bruus, Acoustic Streaming and its Suppression in Inhomogeneous Fluids, Phys. Rev. Lett. 120, 054501 (2018).

[41] See Supplemental Material at http://link.aps.org/supplemental/ 10.1103/PhysRevE.100.023104 for extensions of the results of the following three figures: (1) Fig. 2 turned into one pdf file with 55 subfigures of the acoustic body force $f_{\text {ac }}$ of the double modes $l m 0+l^{\prime} m^{\prime} 0$ with $l, m, l^{\prime}, m^{\prime}=0,1,2,3,4$ (the MATLAB source code is also provided); (2) Fig. 4 turned into five animated gif files of the pressure $p_{1}$ in $3 \mathrm{D}$ and as line plots of the double modes $200+020,400+040$, and $600+060$ both for the wide $\frac{1}{2} L^{\star}$-width and the narrow $\frac{1}{12} L^{\star}$-width $G_{1}$ actuation; and (3) Fig. 8 recalculated using the narrow $\frac{1}{12} L^{\star}$-width $G_{1}$ actuation.

[42] P. Hahn and J. Dual, A numerically efficient damping model for acoustic resonances in microfluidic cavities, Phys. Fluids 27, 062005 (2015).

[43] J. Lei, M. Hill, and P. Glynne-Jones, Numerical simulation of $3 \mathrm{D}$ boundary-driven acoustic streaming in microfluidic devices, Lab Chip 14, 532 (2014).

[44] N. R. Skov, J. S. Bach, B. G. Winckelmann, and H. Bruus, 3D modeling of acoustofluidics in a liquid-filled cavity including streaming, viscous boundary layers, surrounding solids, and a piezoelectric transducer, AIMS Math. 4, 99 (2019).

[45] P. B. Muller and H. Bruus, Numerical study of thermoviscous effects in ultrasound-induced acoustic streaming in microchannels, Phys. Rev. E 90, 043016 (2014).

[46] A. S. Dukhin and P. J. Goetz, Bulk viscosity and compressibility measurement using acoustic spectroscopy, J. Chem. Phys. 130, 124519 (2009).

[47] M. W. H. Ley and H. Bruus, Continuum modeling of hydrodynamic particle-particle interactions in microfluidic highconcentration suspensions, Lab Chip 16, 1178 (2016).

[48] COMSOL MULTIPHYSICS 5.4, http://www.comsol.com.

[49] J. T. Karlsen, P. Augustsson, and H. Bruus, Acoustic Force Density Acting on Inhomogeneous Fluids in Acoustic Fields, Phys. Rev. Lett. 117, 114504 (2016). 